University of Nebraska - Lincoln

DigitalCommons@University of Nebraska - Lincoln

2008

\title{
Enhanced Polymer Grafting from Multiwalled Carbon Nanotubes through Living Anionic Surface-Initiated Polymerization
}

Georgios Sakellariou

Haining Ji

Jimmy W. Mays

Durairaj Baskaran

Follow this and additional works at: https://digitalcommons.unl.edu/usdoepub

Part of the Bioresource and Agricultural Engineering Commons

Sakellariou, Georgios; Ji, Haining; Mays, Jimmy W.; and Baskaran, Durairaj, "Enhanced Polymer Grafting from Multiwalled Carbon Nanotubes through Living Anionic Surface-Initiated Polymerization" (2008). US Department of Energy Publications. 61.

https://digitalcommons.unl.edu/usdoepub/61

This Article is brought to you for free and open access by the U.S. Department of Energy at DigitalCommons@University of Nebraska - Lincoln. It has been accepted for inclusion in US Department of Energy Publications by an authorized administrator of DigitalCommons@University of Nebraska - Lincoln. 


\title{
Enhanced Polymer Grafting from Multiwalled Carbon Nanotubes through Living Anionic Surface-Initiated Polymerization
}

\author{
Georgios Sakellariou, ${ }^{\dagger}$ Haining Ji ${ }^{\dagger}{ }^{\prime}$ Jimmy W. Mays, ${ }^{\dagger}, \#$ and Durairaj Baskaran ${ }^{*}{ }^{\dagger}$ \\ Department of Chemistry, University of Tennessee, 552 Buehler Hall, Knoxville, Tennessee 37996, and \\ Chemical Sciences Division, Oak Ridge National Laboratory, Oak Ridge, Tennessee 37831
}

Received May 27, 2008. Revised Manuscript Received July 14, 2008

\begin{abstract}
Anionic surface-initiated polymerization of ethylene oxide and styrene has been performed using multiwalled carbon nanotubes (MWNTs) functionalized with anionic initiators. The surface of MWNTs was modified via covalent attachment of precursor anions such as 4-hydroxyethyl benzocyclobutene (BCBEO) and 1-benzocyclobutene-1'-phenylethylene (BCB-PE) through Diels-Alder cycloaddition at 235 ${ }^{\circ} \mathrm{C}$. Surface-functionalized MWNTs- $g$-(BCB-EO) ${ }_{n}$ and MWNTs- $g$-(BCB-PE) ${ }_{n}$ with 23 and 54 wt \% precursor initiators, respectively, were used for the polymerizations. Alkoxide anion on the surface of MWNTs- $g$-(BCB-EO) $)_{n}$ was generated through reaction with potassium triphenylmethane for the polymerization of ethylene oxide in tetrahydrofuran and phenyl substituted alkyllithium was generated from the surface of MWNTs- $g$-(BCB-PE) $n$ using sec-butyllithium for the polymerization of styrene in benzene. In both cases, the initiation was found to be very slow because of the heterogeneous reaction medium. However, the MWNTs gradually dispersed in the reaction medium during the polymerization. A pale green color was noticed in the case of ethylene oxide polymerization and the color of initiator as well as the propagating anions was not discernible visually in styrene polymerization. Polymer grafted nanocomposites, MWNTs- $g$-(BCB-PEO $)_{n}$ and MWNTs- $g$-(BCB-PS $)_{n}$ containing a very high percentage of hairy polymer with a small fraction of MWNTs $(<1 \mathrm{wt} \%)$ were obtained. The conversion of ethylene oxide and the weight percent of PEO on the surface of the MWNTs increased with increasing reaction time indicating a controlled polymerization. The polymer-grafted MWNTs were characterized using FTIR, ${ }^{1} \mathrm{H}$ NMR, Raman spectroscopy, differential scanning calorimetry, thermogravimetric analysis, and transmission electron microscopy (TEM). Size exclusion chromatography of the polymer grafted MWNTs revealed broad molecular weight distributions $\left(1.3<M_{\mathrm{w}} / M_{\mathrm{n}}<1.8\right)$ indicating the presence of different sizes of polymer nanocomposites. The TEM images showed the presence of thick layers of polymer up to $30 \mathrm{~nm}$ around the MWNTs. The living nature of the growing polystyryllithium was used to produce diblock copolymer grafts using sequential polymerization of isoprene on the surface of MWNTs.
\end{abstract}

\section{Introduction}

One of the potential applications of carbon nanotubes (CNTs) is their use as nanofibers in designing novel ultrahigh strength polymer composites. ${ }^{1-3}$ A stumbling block in developing polymer-carbon nanotube composite is the difficulty in dispersing CNTs and controlling their orientation in a polymer matrix. Homogeneous dispersion of CNTs will enhance the overall performance of polymer-carbon nanotube composites. $^{2}$ A good interfacial adhesion between CNTs and polymer is essential for load transfer in composite application. For the development of ultrahigh strength polymer-carbon nanotube composites, it is necessary to covalently graft highmolecular-weight polymers on the surfaces of CNTs. Polymer chains with high molecular weight grafted to the CNTs' surface would significantly reduce the interfacial energy in

\footnotetext{
* Corresponding author. E-mail: baskaran@utk.edu.

${ }^{\dagger}$ University of Tennessee.

\# Oak Ridge National Laboratory.

(1) Dresselhaus, M. S.; Avouris, P. Introduction to Carbon Materials Research. In Carbon Nanotubes; Dresselhaus, M. S., Dresselhaus, G., Avouris, P., Eds.; Topics in Applied Physics; Springer-Verlag: Berlin, 2001; Vol. 80, pp 1-9.

(2) Ajayan, P. M. Chem. Rev. 1999, 99, 1787-1799.

(3) Ajayan, P. M.; Schadler, L. S.; Giannaris, C.; Rubio, A. Adv Mater. 2000, 12 (10), 750-753.
}

the composite. Thus, it is desirable to graft high molecular weight polymers on the surface of CNTs for efficient entanglement with the matrix polymer.

Several strategies describing covalent and noncovalent functionalizations of CNTs have been reported, which improved the chemical compatibility and the dissolution properties of the CNTs. ${ }^{4-8}$ Surface modification using small molecules has successfully circumvented the aggregation problem of CNTs and enabled dissolution of CNTs in organic media. ${ }^{4,9-14}$ The covalent functionalization of organic mol-

(4) Chen, J.; Hamon, M. A.; Hu, H.; Chen, Y.; Rao, A. M.; Eklund, P. C.; Haddon, R. C. Science 1998, 282, 95-98.

(5) Banerjee, S.; Kahn, M. G. C.; Wong, S. S. Chem.-Eur. J. 2003, 9, 1898-1908.

(6) Dyke, C. A.; Tour, J. M. Chem.-Eur. J. 2004, 10, 812-817.

(7) Baskaran, D. Carbon: Nanotubes. In Encyclopedia of Inorganic Chemistry, 2nd ed.; King, B. R., Ed.; John Wiley \& Sons: Chichester, U.K., 2005; Vol. II, pp 730-764.

(8) Baskaran, D.; Mays, J. W.; Bratcher, M. S. Chem. Mater. 2005, 17, 3389-3397.

(9) Chen, J.; Rao, A. M.; Lyuksyutov, S.; Itkis, M. E.; Hamon, M. A.; Hu, H.; Cohn, R. W.; Eklund, P. C.; Colbert, D. T.; Smalley, R. E.; Haddon, R. C. J. Phys. Chem. B 2001, 105, 2525-2528.

(10) Sun, Y.-P.; Fu, K.; Lin, Y.; Huang, W. Acc. Chem. Res. 2002, 35 (12), 1096-1104.

(11) Qin, S.; Qin, D.; Ford, W. T.; Resasco, D. E.; Herrera, J. E. J. Am. Chem. Soc. 2004, 126 (1), 170-176. 
ecules or polymers is generally done using either "grafting to" or "grafting from" strategies. ${ }^{4,10-13,15,16}$ The "grafting to" strategy mostly exploits nanotube bound carboxylic acid groups in amidation or esterification reactions with amine or hydroxyl containing polymer or organic molecules. However, the availability of acid groups generated through oxidation procedure on the surface and tips of CNTs is limited. ${ }^{17-19}$ Moreover, to introduce a large concentration of acid groups on the surface, CNTs are digested in acid for long periods of time, which drastically shortens the tubes and can damage the $\mathrm{sp}^{2}$ network severely. Another strategy is to directly use the $\mathrm{sp}^{2}$ bonds of CNTs for reaction with fluorine,${ }^{20}$ nitrenes, ${ }^{21}$ carbenes,${ }^{22}$ diazonium salts, ${ }^{23}$ bromomalonates, ${ }^{24}$ and radicals. ${ }^{25}$ Several other reactions using Vaska's complex, ${ }^{26}$ Wilkinson's catalyst,${ }^{27}$ and cycloaddition $^{28-32}$ also produce covalent functionalization of CNTs. The addition of small molecules via the above-mentioned reactions is successful and provides different degrees of covalent functionalization depending on the reaction conditions. ${ }^{13}$

On the other hand, covalent grafting of macromolecules onto the surface of CNTs has problems associated with adsorption and wrapping. ${ }^{8,33,34}$ We have shown in our previous work that grafting high molecular weight polymer using the "grafting to" approach is inefficient and the level of grafting is inversely proportional to the molecular weight of polymer because of unfavorable conformational entropy upon grafting. ${ }^{8,15,34}$ A substantial amount of polymer used

(12) Baskaran, D.; Mays, J. W.; Bratcher, M. S. Angew. Chem., Int. Ed. 2004, 43 (16), 2138-2142.

(13) Bahr, J. L.; Tour, J. M. Chem. Mater. 2001, 13, 3823.

(14) Dyke, C. A.; Tour, J. M. J. Am. Chem. Soc. 2003, 125, 1156-1157.

(15) Baskaran, D.; Sakellariou, G.; Mays, J. W.; Bratcher, M. S. J. Nanosci. Nanotechnol 2007, 7 (5), 1560-1567.

(16) Yao, Z.; Braidy, N.; Botton, G. A.; Adronov, A. J. Am. Chem. Soc. 2003, 125, 16015-16024.

(17) Satishkumar, B. C.; Govindaraj, A.; Mofokeng, J.; Subbana, G. N.; Rao, C. N. R. J. Phys. B: At. Mol. Phys. 1996, 29, 4925-4934.

(18) Hu, H.; Bhowmik, P.; Zhao, B.; Hamon, M. A.; Itkis, M. E.; Haddon, R. C. Chem. Phys. Lett. 2001, 345, 25-28.

(19) Hamon, M. A.; Hu, H.; Bhowmik, P.; Niyogi, S.; Zhao, B.; Itkis, M. E.; Haddon, R. C. Chem. Phys. Lett. 2001, 347, 8-12.

(20) Mickelson, E. T.; Huffman, C. B.; Rinzler, A.; Smalley, R. E.; Hauge, R. H.; Margrave, J. L. Chem. Phys. Lett. 1998, 296, 188-194.

(21) Holzinger, M.; Abraham, J.; Whelan, P.; Graupner, R.; Ley, L.; Hennrich, F.; Kappes, M.; Hirsch, A. J. Am. Chem. Soc. 2003, 125, $8566-8580$.

(22) Holzinger, M.; Vostrowsky, O.; Hirsch, A.; Hennrich, F.; Kappes, M.; Weiss, R.; Jellen, F. Angew. Chem., Int. Ed. 2001, 40 (21), 4002.

(23) Bahr, J. L.; Yang, J.; Kosynkin, D. V.; Bronikowski, M. J.; Smalley, R. E.; Tour, J. M. J. Am. Chem. Soc. 2001, 123, 6536-6542.

(24) Coleman, K. S.; Bailey, S. R.; Fogden, S.; Green, M. L. H. J. Am. Chem. Soc. 2003, 125, 8722-8723.

(25) Umek, P.; Seo, J. W.; Hernadi, K.; Mrzel, A.; Pechy, P.; Mihailovic, D. D.; Forro, L. Chem. Mater. 2003, 15, 4751-4755.

(26) Banerjee, S.; Wong, S. S. Nano Lett. 2002, 2 (1), 49-53.

(27) Banerjee, S.; Wong, S. S. J. Am. Chem. Soc. 2002, 124, 8940-8948.

(28) Georgakilas, V.; Kordatos, K.; Prato, M.; Guldi, D. M.; Holzinger, M.; Hirsch, A. J. Am. Chem. Soc. 2002, 124 (5), 2002.

(29) Georgakilas, V.; Tagmatarchis, N.; Pantarotto, D.; Bianco, A.; Briand, J.-P.; Prato, M. Chem. Commun. 2002, 3050.

(30) Delgado, J. L.; Cruz, P. d. 1.; Langa, F.; Urbina, A.; Casado, J.; Navarrete, J. T. L. Chem. Commun. 2004, 1734-1735.

(31) Medard-Moyon, C.; Dumas, F.; Doris, E.; Mioskowski, C. J. Am. Chem. Soc. 2006, 128, 14764.

(32) Sakellariou, G.; Ji, H.; Mays, J. W.; Hadjichristidis, N.; Baskaran, D. Chem. Mater. 2007, 19, 6370-6372.

(33) Baskaran, D.; Dunlap, J. R.; Mays, J. W.; Bratcher, M. S. Macromol. Rapid Commun. 2005, 26, 481-486.

(34) Baskaran, D.; Mays, J. W.; Bratcher, M. S. Polymer 2005, 46, 50505057. for the covalent grafting reaction was found to adsorb on the surface of CNTs when grafting reactions are conducted at high solid content under vigorous stirring. During the reaction, a shear induced coating of polymers on CNTs through noncovalent intermolecular interactions occurs, which further complicates quantification of covalent graftingreaction. "8, Although the "grafting to" strategy has been used with polymers in several studies, it appears that it is more suitable for attaching small molecules on the surface of CNTs. ${ }^{8}$

To overcome the entropy penalty associated with grafting of high molecular weight polymer, we and others have adopted a "grafting from" strategy using surface initiated polymerization (SIP) to grow polymers from CNTs. ${ }^{1,12,16,33,35-37}$ In most cases, the CNTs were functionalized with 2-hydroxyethyl-2bromopropionate, an initiator for atom transfer radical polymerization (ATRP), and used for the polymerization of styrene and methyl methacrylate. In our hands, we could grow up to $70 \mathrm{wt} \%$ of homopolymer or block copolymer on the surface of multiwalled carbon nanotubes (MWNTs) using this approach. ${ }^{12}$ Although one would expect that SIP using ATRP provides an opportunity to grow polymer in a controlled manner, high-molecular-weight polymer growth was found to be difficult. This could be attributed to the fact that a very low concentration of initiator $\left(\sim 1 \times 10^{-4}\right.$ to $1 \times 10^{-5} \mathrm{~mol} / \mathrm{g}$ of carbon) available on the surface of CNTs leading to termination of growing chain-radicals in a heterogeneous ATRP. ${ }^{16}$

An ideal choice of polymerization conditions for growing polymer chains from CNTs is the one that should be free from side reactions and can be operated in a controlled manner, even at a low initiator concentration as well as under heterogeneous polymerization conditions. This prompted us to turn our attention to living anionic polymerization, which can produce high-molecular-weight polymers in a controlled manner even at a very low concentration of initiator. Recently, surface-initiated anionic polymerization from single-walled carbon nanotubes (SWNTs) has been attempted using anions generated on the surface via sec-butyllithium ( eec-BuLi) addition to the $\mathrm{sp}^{2}$ carbons, and styrene was polymerized in the presence of excess initiator to synthesis polystyrene nanocomposite. ${ }^{38}$ However, the grafting efficiency was low $(\sim 10$ wt $\%$ ) because of low efficiency of anion generation on the surface. Thus, the challenge remains to grow high-molecular-weight polymers in a controlled manner without disturbing the structural integrity of CNTs. Herein, we describe a robust method to grow high molecular weight poly(ethylene oxide) and polystyrene from the surfaces of MWNTs using living anionic polymerization. Controlled covalent functionalization has been used to attach 4-hydroxyethyl benzocyclobutene (BCB-EO) and 1-benzocyclobutene-1'-phenylethylene (BCB-PE) initiator moieties on the surface of MWNTs for ethylene oxide and styrene

(35) Kong, H.; Gao, C.; Yan, D. J. Am. Chem. Soc. 2004, 126, 412-413.

(36) Xu, Y.; Gao, C.; Kong, H.; Yan, D.; Jin, Y. Z.; Watts, P. C. P. Macromolecules 2004, 37, 8846-8853.

(37) Gomez, F. J.; Chen, R. J.; Wang, D.; Waymouth, R. M.; Dai, H. Chem. Commun. 2003, 190-191.

(38) Viswanathan, G.; Chakrapani, N.; Yang, H.; Wei, B.; Chung, H.; Cho, K.; Ryu, C. Y.; Ajayan, P. M. J. Am. Chem. Soc. 2003, 125 (31), 9258-9259. 
Scheme 1. Synthesis of 4-Hydroxyethyl Benzocyclobutene (BCB-EO) and 1-Benzocyclobutene-1'-phenylethylene (BCB-PE) Using Grignard Reagent of 4-Bromobenzocyclobutene with Ethylene Oxide and Acetophenone, Respectively

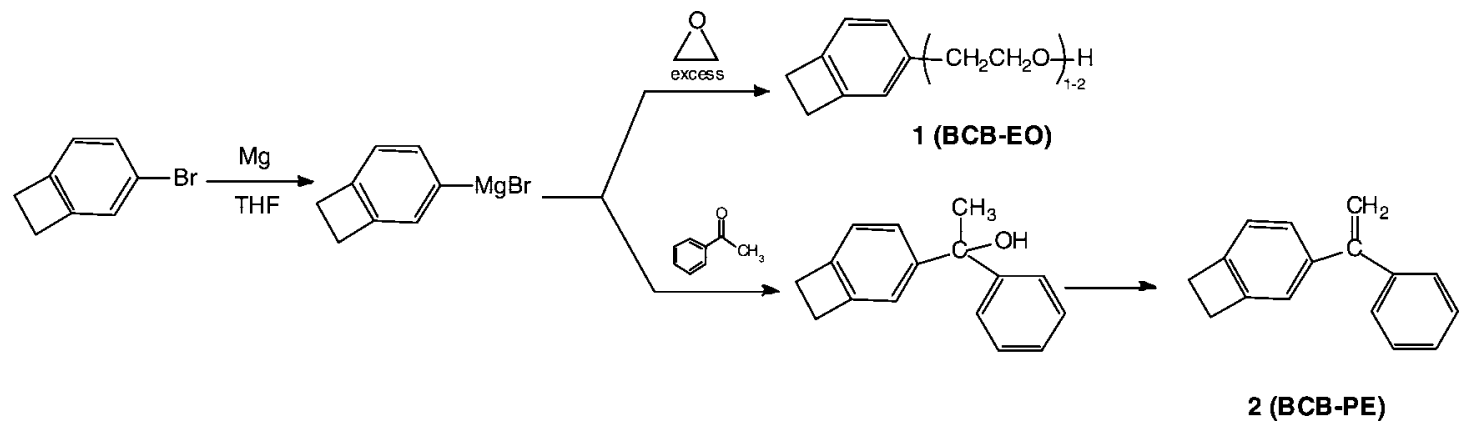

polymerizations, respectively. The living nature of the polymerizations has been confirmed through kinetic studies and block copolymer formation.

\section{Experimental Section}

Materials. The MWNTs were provided by NanoLab (Watertown, MA). The MWNTs were obtained through the chemical vapor deposition method and were purified with HF wash to remove residual catalyst. It was found that the MWNTs yielded a small quantity of iron or iron oxide particles as residue $(\sim 4-5 \mathrm{wt} \%)$ in the thermogravimetric analysis (TGA). The acid groups present on the MWNTs were removed by annealing the tubes at $250{ }^{\circ} \mathrm{C}$ for $2 \mathrm{~h}$ under air followed by heating to $450{ }^{\circ} \mathrm{C}$ for $2 \mathrm{~h}$ under $\mathrm{N}_{2}$. 4-Bromobenzocyclobutene (BCB-Br, 97\%) was kindly donated by Dow Chemical (USA). Acetophenone, styrene, isoprene, ethylene oxide, tetradecane, benzene, $n$-hexane, and magnesium turnings (99.99\%) were purchased from Aldrich, USA. sec-BuLi was synthesized in-house. ${ }^{39,40}$ Monomers and solvents were purified according to standard procedures. ${ }^{39}$ Ethylene oxide (EO) was distilled over a small amount of $n$-BuLi. Magnesium turnings were washed with distilled tetrahydrofurn (THF) and dried under a vacuum.

Synthesis of 4-Hydroxyethyl Benzocyclobutene (BCB-EO) (1). To a $500 \mathrm{~mL}$ flask was added $50 \mathrm{~mL}$ of dry THF, Mg turnings $(2.9 \mathrm{~g})$, and 1,2-dibromoethane (4-5 drops). The reaction mixture was then heated under reflux for $15 \mathrm{~min}$. 4-Bromobenzocyclobutene $(20 \mathrm{~g})$ in $25 \mathrm{~mL}$ of THF was added via a dropping funnel to form the Grignard reagent (Scheme 1). After addition and rinsing the funnel with $25 \mathrm{~mL}$ of dry THF, the reaction mixture was heated for an additional 45 min under reflux to give a green-brown solution. The mixture was then cooled to $0{ }^{\circ} \mathrm{C}$; ethylene oxide in dry THF was added dropwise to the solution, and the reaction mixture was heated under reflux for $15 \mathrm{~min}$. The reaction mixture was poured onto $150 \mathrm{~g}$ of ice, acidified to $\mathrm{pH} 4$, and neutralized with saturated $\mathrm{NaHCO}_{3}$ solution. The crude product was extracted with ethyl acetate. 4-Hydroxyethyl benzocyclobutene was obtained in $75 \%$ yield. ${ }^{1} \mathrm{H}$ NMR of the product showed that it contains $\sim 40 \%$ dimerized ethylene oxide units. ${ }^{1} \mathrm{H}$ NMR $\left(\mathrm{CDCl}_{3}\right): \delta 2.80(2 \mathrm{H}, \mathrm{t})$, $3.15(4 \mathrm{H}, \mathrm{s}), 3.78(2 \mathrm{H}, \mathrm{t})$, and $6.90-7.10(3 \mathrm{H}, \mathrm{m})$.

Synthesis of 1-Benzocyclobutene-1'-phenylethylene (BCB-PE) (2). The Grignard reagent prepared from 4-bromobenzocyclobutene was used for the reaction with acetophenone (Scheme 1). Accordingly, a required amount of acetophenone $(25.2 \mathrm{~g}, 210 \mathrm{mmol})$ in $50 \mathrm{~mL}$ of dry THF was added dropwise to the Grignard reagent as prepared above at $0{ }^{\circ} \mathrm{C}$ and the reaction mixture was heated under

(39) Hadjichristidis, N.; Iatrou, H.; Pispas, S.; Pitsikalis, M. J. Polym. Sci., Part A: Polym. Chem. 2000, 38, 3211-3234.

(40) Uhrig, D.; Mays, J. W. J. Polym. Sci., Part A: Polym. Chem. 2005, 43, 6179-6222. reflux for $15 \mathrm{~min}$. The reaction mixture was poured into $150 \mathrm{~g}$ of ice, acidified to $\mathrm{pH} 4$, and neutralized with saturated $\mathrm{NaHCO}_{3}$ solution. The dark yellow-green residue was dissolved in acetic anhydride $(150 \mathrm{~mL})$ and a small amount of $\mathrm{H}_{2} \mathrm{SO}_{4}$ (2 drops) was added. This mixture was heated to reflux at $100{ }^{\circ} \mathrm{C}$, for $21 / 2 \mathrm{~h}$. After cooling, the yellow-green product was collected and purified by column chromatography with a solvent mixture of hexanetoluene. 1-Benzocyclobutene-1'-phenylethylene (BCB-DPE) was obtained in $60 \%$ yield. ${ }^{1} \mathrm{H}$ NMR $\left(\mathrm{CDCl}_{3}\right): 3.12(4 \mathrm{H}, \mathrm{s}), 5.24(2 \mathrm{H}$, $\mathrm{s})$, and $7.16(8 \mathrm{H}, \mathrm{m})$.

Diels-Alder Reactions of Substituted Benzocylobutenes 1 and $\mathbf{2}$ with MWNTs. The precursor initiator moieties such as $\mathbf{1}$ and $\mathbf{2}$ were covalently attached to the MWNTs using Diels-Alder [4+ 2] cycloaddition. ${ }^{32}$ In a typical reaction, $100 \mathrm{mg}$ of annealed MWNTs and $10 \mathrm{~mL}$ of tetradecane were placed in a glass reactor having an inlet and an outlet for argon. The mixture was purged with argon for $10 \mathrm{~min}$ and then heated to $235^{\circ} \mathrm{C}$ under an argon atmosphere with stirring. One milliliter of BCB-EO (1) or BCB$\mathrm{PE}$ (2) was added dropwise to the mixture via a peristaltic pump at a rate of $1.25 \mathrm{~mL} / \mathrm{h}$ with vigorous stirring under argon. After the addition, the reaction mixture was stirred for a desired period of time and then cooled to room temperature. The substituted benzocyclobutene grafted MWNTs (3, MWNTs- $g$-(BCB-EO) ${ }_{n}$ and 4, MWNTs- $g$-(BCB-PE) $)_{n}$ ) were collected by washing with copious amount of THF and filtered using a Teflon membrane $(0.2 \mu \mathrm{m})$. The washing was repeated until the filtrate showed an absence of 1 or 2 using thin-layer chromatography (TLC). The side-wallfunctionalized MWNTs- $g$-(BCB-EO $)_{n}$ and MWNTs- $g$-(BCB-PE) ${ }_{n}$ were dried at $60{ }^{\circ} \mathrm{C}$ for $5 \mathrm{~h}$ under a vacuum.

Surface-Initiated Living Anionic Polymerization of Ethylene Oxide Using 3, MWNTs-g-(BCB-EO) $)_{n}$. Surface-initiated anionic polymerization of ethylene oxide from the hydroxyl groups of the MWNTs- $g$-(BCB-EO) ${ }_{n}$ was performed in a specially designed glassreactor under vacuum (Figure 1a). First, a required amount of $\mathbf{3}$ bearing $23 \mathrm{wt} \%$ BCB-EO $\left(10 \mathrm{mg},[\mathrm{I}]=2.3 \times 10^{-5} \mathrm{~mol}\right)$ was sealed under vacuum in a clean glass ampule containing $15 \mathrm{~mL}$ of purified THF. This ampule was assembled onto the reactor along with other purified reagents such as ethylene oxide (1.0 g in THF), triphenylmethyl potassium (TPM, $2.0 \times 10^{-5} \mathrm{~mol}$ in THF), and methanol $(2 \mathrm{~mL})$. The reactor also had a purge section $(100 \mathrm{~mL}$ flask) with a rubber septum inlet. The reactor was docked to a high vacuum line, and the entire reactor was evacuated to attain high vacuum (ca. $1 \times 10^{-6}$ torr). A small amount of $n$-BuLi (1.3 M in hexane) and 1,1'-diphenylethylene (DPE, a few drops) were then syringed into the purge section of the reactor under static vacuum, and the septum portion was removed by sealing the glass constriction after washing with $n$-hexane. The reaction of $n$-BuLi and DPE gave a red-colored adduct anion (1,1-diphenylhexyllithium, DPHLi), which was used as an indicator for cleaning the reactor under a vacuum. About $50 \mathrm{~mL}$ of purified THF was then distilled into the 


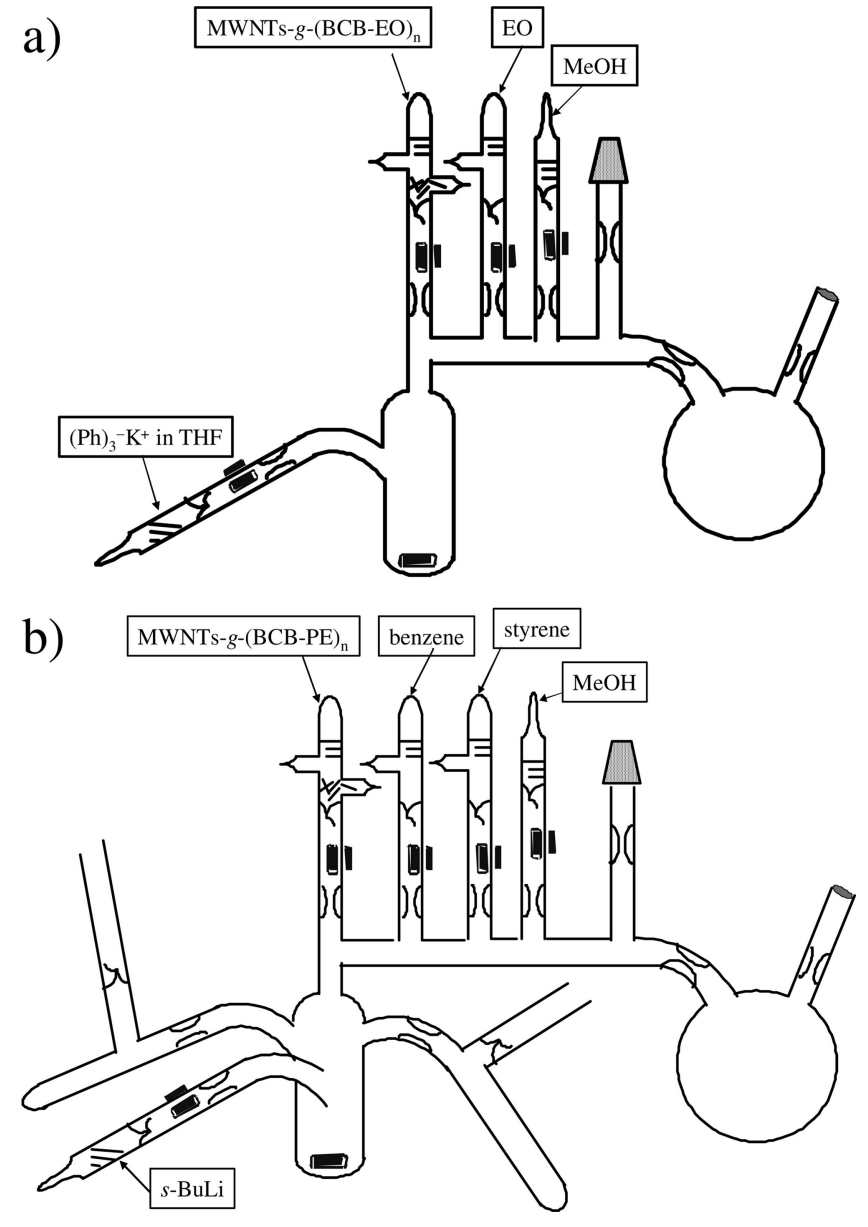

Figure 1. All-glass reactors used for the surface initiated anionic polymerization of (a) ethylene oxide and (b) styrene from MWNTs- $g$-(BCB-EO) ${ }_{n}$ and MWNTs- $g$-(BCB-PE) ${ }_{n}$, respectively.

reactor and the entire reactor was sealed off from the vacuum line. The solution of DPHLi in THF was used to rinse the reactor to quench impurities present on the glass surface. Subsequently, the internal walls of the reactor were washed thoroughly by condensing pure THF from the purge section to remove all the excess DPHLi. The required amount of THF was then distilled into the reactor from the purge section containing DPHLi anion solution in THF. The purge section was removed from the reactor under a vacuum through heat-sealing.

The MWNTs- $g$-(BCB-EO $)_{n}$ in THF was introduced by opening the break-seal into the main chamber of the reactor. The red color of triphenylmethyl potassium (TPM) in THF was used as an indicator to determine the extent of metalation while generating alkoxide anions on the surface of MWNTs. Accordingly, the TPM ampule was opened and the reactor was tilted so that only a small quantity of TPM dropped into the reactor containing MWNTs- $g$ $(\mathrm{BCB}-\mathrm{EO})_{n}$ in THF. The red color of the TPM anions upon continuous stirring slowly disappeared and became pale green. The disappearance of the red color indicated abstraction of hydrogen from the surface hydroxyl groups of MWNTs- $g$-(BCB-EO) $)_{n}$.

Because alkoxide anions of this type do not exhibit a characteristic color, the formation of a pale green color suggests that the newly generated alkoxides on the surface may have strong interactions with $\pi$ electrons of the MWNTs. The titration was continued until the rate of disappearance of the red color was very slow. After several titration steps, a faint red color of the TPM anions remained for more than two hours indicating a majority of hydroxyl groups on the MWNTs was converted into potassium alkoxide anions. After complete disappearance of the red color of
TPM anions in the solution, the ampule with the remaining TPM solution was removed from the system and the polymerization of ethylene oxide was started by mixing ethylene oxide into the heterogeneous solution of MWNTs bearing surface alkoxide initiators. The polymerization of ethylene oxide was conducted at $50{ }^{\circ} \mathrm{C}$ in a heterogeneous medium. The reaction was allowed to proceed for several hours under stirring. During the polymerization, it was noticed that the viscosity of the heterogeneous solution slowly increased and the MWNTs gradually dispersed under fast stirring. After a few hours, the polymerization medium became homogeneous and dark black in color. The reaction was terminated by adding a small amount of methanol $(2 \mathrm{~mL})$. The viscous black colored polymerization solution could not be filtered through a 0.4 $\mu \mathrm{m}$ Teflon membrane at high concentration. The PEO grafted MWNTs were recovered by precipitation in cold $n$-hexane. In all the polymerizations, the yield was more than $100 \%$ on the basis of MWNTs because of PEO growth. The conversion of EO was determined gravimetrically.

A control experiment was also performed in an identical manner in the presence of pristine MWNTs. In this case, the red color persisted upon addition of first drop of TPM anions because of the absence of hydroxyl groups on the surface of MWNTs. A known quantity of ethylene oxide was added into this solution to start the polymerization. The red color of TPM anions changed immediately to pale green, indicating initiation of ethylene oxide. The polymerization was continued and it was found that the MWNTs did not disperse in the polymerization medium. This clearly suggests that the polymerization proceeded in the solution without incorporating MWNTs. The reaction was terminated with methanol $(2 \mathrm{~mL})$ after $24 \mathrm{~h}$ and the PEO generated in the solution was filtered using 0.4 $\mu \mathrm{m}$ Teflon membrane to recover the MWNTs. The PEO was precipitated in cold $n$-hexane as colorless powder, which confirms the absence of MWNTs.

Surface-Initiated Living Anionic Polymerization of Styrene Using 4, MWNTs-g-(BCB-PE) $)_{n}$. A typical surface-initiated anionic polymerization of styrene was performed in a special reactor shown in Figure 1b. An ampule containing MWNTs- $g$-(BCB-PE) $)_{n}(15 \mathrm{mg}$, $\left.[\mathrm{I}]=3.93 \times 10^{-5} \mathrm{~mol}, 54 \mathrm{wt} \% \mathrm{BCB}-\mathrm{PE}\right)$ in $15 \mathrm{~mL}$ of purified $n$-hexane was attached to the reactor along with other ampules having purified reagents such as styrene $(1.8 \mathrm{~g})$, benzene $(20 \mathrm{~mL})$, sec-BuLi $\left(4.0 \times 10^{-5} \mathrm{~mol}\right.$ in $n$-hexane), and methanol $(2 \mathrm{~mL})$. The reactor also had a purge section $(100 \mathrm{~mL}$ flask) with a rubber septum inlet. The reactor was docked to a high vacuum line and the entire reactor was evacuated to attain high vacuum. Then, a small amount of $n$-BuLi (1.3 M in hexane) was syringed into the purge section of the reactor under static vacuum and the septum portion was removed by sealing the glass constriction after washing with $n$-hexane. About $50 \mathrm{~mL}$ of purified benzene was then distilled into the reactor and the entire reactor was sealed off from the vacuum line. After rinsing the entire reactor with sec-BuLi solution in benzene, the reactor was rinsed through distillation. A known quantity of pure benzene was distilled into the main reactor from the purge section containing collected remaining sec-BuLi. Subsequently, the purge section was removed from the reactor through heat-sealing at the glass constriction.

The MWNTs- $g$-(BCB-PE) $)_{n}$ and $s e c$-BuLi were introduced by opening the break-seals of the respective ampules. Sec-BuLi was allowed to react with the surface BCB-PE groups of MWNTs to generate carbanions under stirring at room temperature for $12 \mathrm{~h}$. The orange color of a typical delocalized phenyl substituted anions in benzene was not discernible on the surface of MWNTs. Then, the carbon nanotubes were allowed to settle and the supernatant solution containing excess amounts of sec-BuLi was decanted into a sidearm. The solvent was back-distilled into the chamber 
containing carbon nanotubes to wash adsorbed sec-BuLi from the tubes. This washing procedure was repeated more than 10 times to thoroughly remove any traces of free $s e c$-BuLi on the MWNTs. Finally, the sidearm with the collected sec-BuLi was removed by heat-sealing. The ampule containing $20 \mathrm{~mL}$ of benzene was then ruptured. After stirring the benzene solution of the functionalized MWNTs for half an hour, the carbon nanotubes were allowed to settle. The absence of free sec-BuLi in the polymerization solution was confirmed through testing for styrene initiation. Accordingly, a small amount of supernatant benzene $(2 \mathrm{~mL})$ was taken from the reactor by decantation in to a second sidearm. This benzene solution was attached to another reactor, wherein a small amount of styrene $(1 \mathrm{~mL})$ was mixed under a vacuum in order to check the presence of sec-BuLi. There was no development of orange color, which indicated absence of free initiator in the polymerization solution.

Subsequently, styrene $(1.8 \mathrm{mg})$ was added into the main reactor containing MWNTs with surface anions to start the surface initiated anionic polymerization. The reaction was performed for several hours under stirring. In contrast to normal styrene polymerization, the polymerization solution did not develop an orange color. However, the viscosity of the solution gradually increased during the polymerization, indicating progress of the polymerization. Reactions carried out for a short time (45 and $60 \mathrm{~min}$ ) had a small amount of MWNTs- $g$-(BCB-PE) ${ }_{n}$ settling at the bottom of the reactor during the polymerization. This indicates that the initiation from the MWNTs surface is very slow and the propagation of initiated chains is fast. However, the MWNTs gradually dispersed during the polymerization over $1 \mathrm{~h}$ and the entire solution became homogeneously black in color. The reaction was terminated with degassed methanol. A similar procedure was used for PS- $b$-PI diblock copolymerization. The second monomer, isoprene, was added after complete conversion of styrene $(24 \mathrm{~h})$. The polymerization of isoprene was conducted for $18 \mathrm{~h}$ and terminated with methanol $(2 \mathrm{~mL})$. The polymer grafted MWNTs- $g$-(BCB-PS $)_{n}$ and MWNTs- $g$-(BCB-PS- $b$-PI $)_{n}$ were recovered by precipitation in methanol and appeared gray in color. The diblock grafted MWNTs were obtained in quantitative yield with respect to monomer feed.

Characterization. Thermogravimetric analysis (TGA) was performed using a TA Instruments TGA Q500 in air and nitrogen from 50 to $900{ }^{\circ} \mathrm{C}\left(10{ }^{\circ} \mathrm{C} / \mathrm{min}\right)$. FT-IR spectra were recorded using a Bio-Red Win-IR-Pro instrument with a resolution of $2 \mathrm{~cm}^{-1}$. $\mathrm{KBr}$ was used to prepare sample pellets. Tabletop ultrasonic cleaner (FS$20 \mathrm{H}$, Fisher Scientific) operating at $40 \mathrm{kHz}$ with $80 \mathrm{~W}$ was used. Raman spectra were acquired using the samples in solid state in the backscattering mode on the "microstage" of a Dilor XY Raman spectrometer (Instruments S.A., Inc., Edison, NJ). The $514.5 \mathrm{~nm}$ line of a Coherent Innova 200 argon ion laser was used for excitation. The laser output power $100 \mathrm{~mW}$ was attenuated by $\sim 86 \%$ when measured at the sample position. Spectra were recorded over the range of $800-2000 \mathrm{~cm}^{-1}$ for $10-12$ acquisitions (with 20 s/acquisition) and averaged. The spectra were smoothed with a 7 points adjacent averaging smooth function.

Size exclusion chromatography (SEC) (PL-GPC 120) was used to determine molecular weights and polydispersity indices $\left(M_{\mathrm{w}} /\right.$ $M_{\mathrm{n}}$ ) of the polymer samples with respect to polystyrene standards (PSS, Germany). The unit was equipped RI, light scattering 15 and $90^{\circ}$ (Precision Detectors 2040, $\lambda=685 \mathrm{~nm}, 30 \mathrm{~mW}$ ) and differential viscometer (Viscotek 220) detectors. Polystyrene grafted MWNTs were analyzed using Polymer Laboratories $2 \times$ mixed-B SDV gel columns $(30 \mathrm{~cm})$ and THF was used as the mobile phase at a flow rate of $1 \mathrm{~mL} \mathrm{~min}^{-1}$ at $30^{\circ} \mathrm{C}$. Poly(ethylene oxide)grafted MWNTs were analyzed using Polymer Laboratories $2 \times$ mixed Aquagel$\mathrm{OH}$ columns $(30 \mathrm{~cm})$ and water (with $0.02 \%$ sodium azide) was used as mobile phase at a flow rate of $1 \mathrm{~mL} \mathrm{~min}^{-1}$ at $30{ }^{\circ} \mathrm{C}$. PSS
WinGPC software was used to acquire and analyze the chromatograms. In some cases, SEC was performed on a SEC-LS system consisting of a RI, light scattering 15 and $90^{\circ}$ (Precision Detectors $2040, \lambda=685 \mathrm{~nm}, 30 \mathrm{~mW}$ ) and differential viscometer (Viscotek 220) detectors. Although polymer grafted MWNTs samples are not filterable at high concentration during the synthesis, at dilute concentration $(0.5 \mathrm{mg} / \mathrm{mL})$, they pass through $0.4 \mu \mathrm{m}$ Teflon membrane for SEC analysis. Transmission electron microscopy (TEM) analysis was performed using a Hitachi H-800 TEM operating with an accelerating voltage of $100 \mathrm{KV}$. Samples were prepared by dropping an $\sim 5 \mu \mathrm{L}$ solution on a freshly glow discharged carbon film supported by a 400 mesh $\mathrm{Cu}$ grid. ${ }^{1} \mathrm{H}$ NMR spectra were acquired using a $300 \mathrm{MHz}$ Bruker instrument using tetramethylsilane (TMS) as reference and solutions in $\mathrm{CDCl}_{3}$.

\section{Results and Discussion}

Covalent Functionalization of MWNTs with Precursor Anionic Initiators. A successful living anionic polymerization from the surface of MWNTs requires first to covalently attach functional groups which can be used to generate anions for the polymerization. A direct addition of sec-BuLi to generate secondary anions on the surface of CNT was inefficient. ${ }^{38}$ Hence, it is necessary to functionalize MWNTs with potential anion precursors for the polymerization of ethylene oxide and styrene. In general, alkoxides are used for the polymerization of ethylene oxide, whereas alkyl anions are used for the polymerization of styrene. ${ }^{41-43}$ Thus, MWNTs with potential anion precursor functionalities such as, hydroxyl and diphenylethylene are needed for the surface-initiated anionic polymerization of ethylene oxide and styrene, respectively.

To accomplish this task, substituted benzocyclobutenes were chosen to attach anion precursors on the MWNTs using a Diels-Alder $[4+2]$ cycloaddition reaction. ${ }^{32,44}$ As the benzocylobutene ring is susceptible to thermal ring opening at high temperature $\left(>225^{\circ} \mathrm{C}\right)$, it will form an o-quinodimethane diradical intermediate that undergoes cycloaddition to $s p^{2}$ carbons of MWNTs. The advantage of using this cycloaddition reaction for the covalent functionalization of MWNTs is that, it will produce a statistical distribution of functional groups throughout the surface without causing damage to the carbon backbone of the tubes. For this purpose, initiator precursors bearing benzocyclobutenes, such as 4-hydroxyethyl benzocyclobutene and 1-benzocyclobutene1'-phenylethylene, were synthesized (Scheme 1). Grignard reagent generated from 4-bromobenzocyclobutene was used to react with excess ethylene oxide in THF to produce 4-hydroxyethyl benzocyclobutene (1, BCB-EO). Similarly, acetophenone was reacted with the above Grignard reagent to form a tertiary hydroxyl substituted intermediate, which on thermal dehydration produced 1-benzocyclobutene-1'phenylethylene (2, BCB-PE). The substituted benzocyclobutenes, 1 and $\mathbf{2}$ were obtained in high yield $(\sim 75 \%)$.

(41) Morton, M. Anionic Polymerization: Principles and Practice; Academic Press: New York, 1983.

(42) Feng, X.-S.; Taton, D.; Chaikof, E. L.; Gnanou, Y. J. Am. Chem. Soc. 2005, 127, 10956-10966.

(43) Quirk, R. P.; Tsai, Y. Macromolecules 2003, 31, 8016-8025.

(44) Lu, X.; Tian, F.; WAng, N.; Zhang, Q. Org. Lett. 2002, 4 (24), 43134315. 


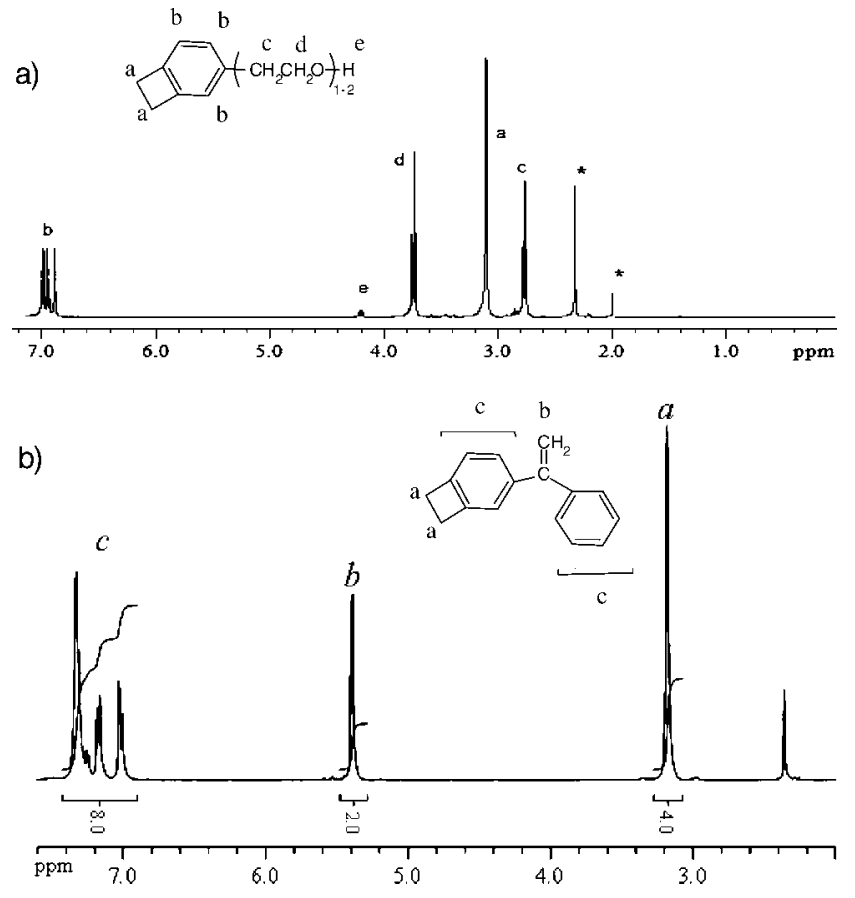

Figure 2. ${ }^{1} \mathrm{H}$ NMR of 4-hydroxyethyl benzocyclobutene (1, a) and 1-benzocyclobutene-1'-phenylethylene $(\mathbf{2}, \mathbf{b})$.

${ }^{1} \mathrm{H}$ NMR of the $\mathbf{1}$ showed presence of characteristic signals at $2.78,3.75$, and $4.2 \mathrm{ppm}$ corresponding to the protons of methylene and hydroxyl groups, respectively (Figure 2a). However, the integration of these signals with either aromatic or cyclobutene groups showed the product contained $60 \%$ monomeric and $40 \%$ dimeric ethylene oxide units. Figure $2 \mathrm{~b}$ shows the ${ }^{1} \mathrm{H}$ NMR of 2 with olefin signal at $5.24 \mathrm{ppm}$ along with aromatic and cyclobutene groups.

The covalent functionalization MWNTs was carried out using [4 2] cycloaddition with 1 and $\mathbf{2}$ (Scheme 2). The purified MWNTs were first subjected to a thermal treatment to remove acid groups present on their surface. This defunctionalization was done using thermal annealing at 450 ${ }^{\circ} \mathrm{C}$ in air for $2 \mathrm{~h}$ and $600{ }^{\circ} \mathrm{C}$ in $\mathrm{N}_{2}$ for $2 \mathrm{~h}$. The covalent functionalization using $\mathbf{1}$ and $\mathbf{2}$ was performed by dropwise addition to MWNTs kept in tetradecane at $235{ }^{\circ} \mathrm{C}$. The temperature of $\mathbf{1}$ and $\mathbf{2}$ was maintained at room temperature until the addition to minimize dimerization and oligomerization before reacting with MWNTs at $235{ }^{\circ} \mathrm{C}$. Table 1 shows the results of covalent functionalization via Diels-Alder adduct formation using 1 and $\mathbf{2}$ with MWNTs. Benzocyclobutene grafted MWNTs (MWNTs- $g$-(BCB-derivative) ${ }_{n}$ ) were recovered from the reaction mixture by filtration using a $0.4 \mu \mathrm{m}$ Teflon membrane. The functionalized MWNTs were thoroughly washed using THF to remove dimer, oligomer, and any remaining $\mathbf{1}$ and $\mathbf{2}$. The washing procedure was continued until the filtrate showed the absence of such products by TLC. The 4-hydroxyethyl benzocyclobutene and 1-benzocyclobutene-1'-phenylethylene functionalized MWNTs such as MWNTs- $g$-(BCB-EO) ${ }_{n}$ (3) and MWNTs- $g$-(BCB$\mathrm{PE})_{n}(4)$, respectively, were dried at $60{ }^{\circ} \mathrm{C}$ for $5 \mathrm{~h}$ under a vacuum and characterized using FT-IR, UV-vis, Raman spectroscopy, and TGA.
The FT-IR spectrum of $\mathbf{3}$ showed characteristic hydroxyl stretching, $\mathrm{C}-\mathrm{H}$ stretching, aromatic ring bending frequencies centered at 3440,2925 , and $1638-1462 \mathrm{~cm}^{-1}$, respectively (Figure 3i). Similarly, the spectrum of $\mathbf{4}$ showed presence of a signal centered at 3100 and $2919 \mathrm{~cm}^{-1}$ corresponding to aromatic and olefin $\mathrm{C}-\mathrm{H}$ functionality (Figure 3ii). A huge band at $\sim 3400 \mathrm{~cm}^{-1}$ is due to the presence of moisture in the sample. Raman spectroscopy of 3 and 4 exhibited tangential (t) and disorder (d) bands at $\sim 1560$ and $1350 \mathrm{~cm}^{-1}$, respectively, similar to pristine MWNTs except that the peak ratio $I_{\mathrm{d}} / I_{\mathrm{t}}$ was slightly higher because of the addition of $\mathrm{sp}^{3}$ carbons on the MWNTs (see the Supporting Information). The functionalized MWNTs 3 and 4 were soluble in $\mathrm{CHCl}_{3}$ after a brief sonication and showed featureless UV-vis absorption spectra that decrease monotonously from 250 to $900 \mathrm{~nm}$.

The amount of grafted $\mathbf{1}$ and $\mathbf{2}$ in $\mathbf{3}$ and $\mathbf{4}$ was determined using TGA. Thermal decomposition of $\mathbf{3}$ in air showed two distinct weight losses at temperatures between 250 and 450 ${ }^{\circ} \mathrm{C}$ and 450 and $650{ }^{\circ} \mathrm{C}$ corresponding to 4-hydroxyethyl benzocyclobutene fragment and MWNTs, respectively (Figure 4i). Similarly, in the case of $\mathbf{4}$, the decomposition of 1-benzocyclobutene- $1^{\prime}$-phenylethylene occurred over temperatures in the range of $250-500{ }^{\circ} \mathrm{C}$ (Figure 4ii). The observed peak maximum decomposition temperature $\left(T_{\mathrm{d}-\text { max }}\right)$ of these initiator fragments on MWNTs is slightly higher than the decomposition of neat $\mathbf{1}$ and $\mathbf{2}$, indicating improved thermal stability due to the presence of MWNTs. The mole $\%$ concentration of $\mathbf{3}$ and $\mathbf{4}$ on the surface of MWNTs was calculated using their weight percent decomposition between 250 and $500{ }^{\circ} \mathrm{C}$ and the molecular weight of benzocylobutenes fragment. It was found that the amount of covalent functionalization through Diels-Alder cycloaddition is dependent on the nature of benzocyclobutene, temperature, and the duration of reaction. The MWNTs with varying degree of covalently functionalized anionic precursors $(1.16<\mathrm{mol}$ $\%<3.45)$ were obtained depending on the reaction conditions (Table 1).

Surface-Initiated Anionic Polymerization of Ethylene Oxide from MWNTs- $g$-(BCB-EO $)_{n}$. The hydroxyl groups of 3, MWNTs- $g$-(BCB-EO) ${ }_{n}$, containing 23 wt $\%$ of 4-hydroxyethyl benzocyclobutene fragments were used for the surface initiated anionic polymerization of ethylene oxide (EO) in THF at $50{ }^{\circ} \mathrm{C}$ (Scheme 3). It is known that EO polymerization can be initiated using alkoxide anion with the potassium counterion. ${ }^{41,42}$ A minimum conversion of $30 \%$ hydroxyl groups into alkoxide anions is sufficient for the initiation of EO as alkoxide anions exist in rapid equilibrium with free hydroxyl groups. ${ }^{42}$ The generation of alkoxide anions and the polymerization of EO were performed using standard all glass-reactor under a high vacuum. Triphenylmethyl potassium (TMP, $2 \times 10^{-3} \mathrm{M}$ ) in THF was used for deprotonation of hydroxyl groups on the surface of MWNTs.

A known quantity of $\mathbf{3}$ was placed in a reactor under a vacuum and the hydroxyl groups on the surface of $\mathrm{MWNT}_{\mathrm{S}^{-}}$ $g$-(BCB-EO $)_{n}$ were converted to alkoxide anions by a slow dropwise addition of TMP in THF at room temperature. The red color of the TMP anion disappeared immediately and a 
Scheme 2. Covalent Attachment of Precursor Benzocyclobutene Derivatives (1 and 2) on the Surface of MWNTs using [4 +2$]$ Cycloaddition

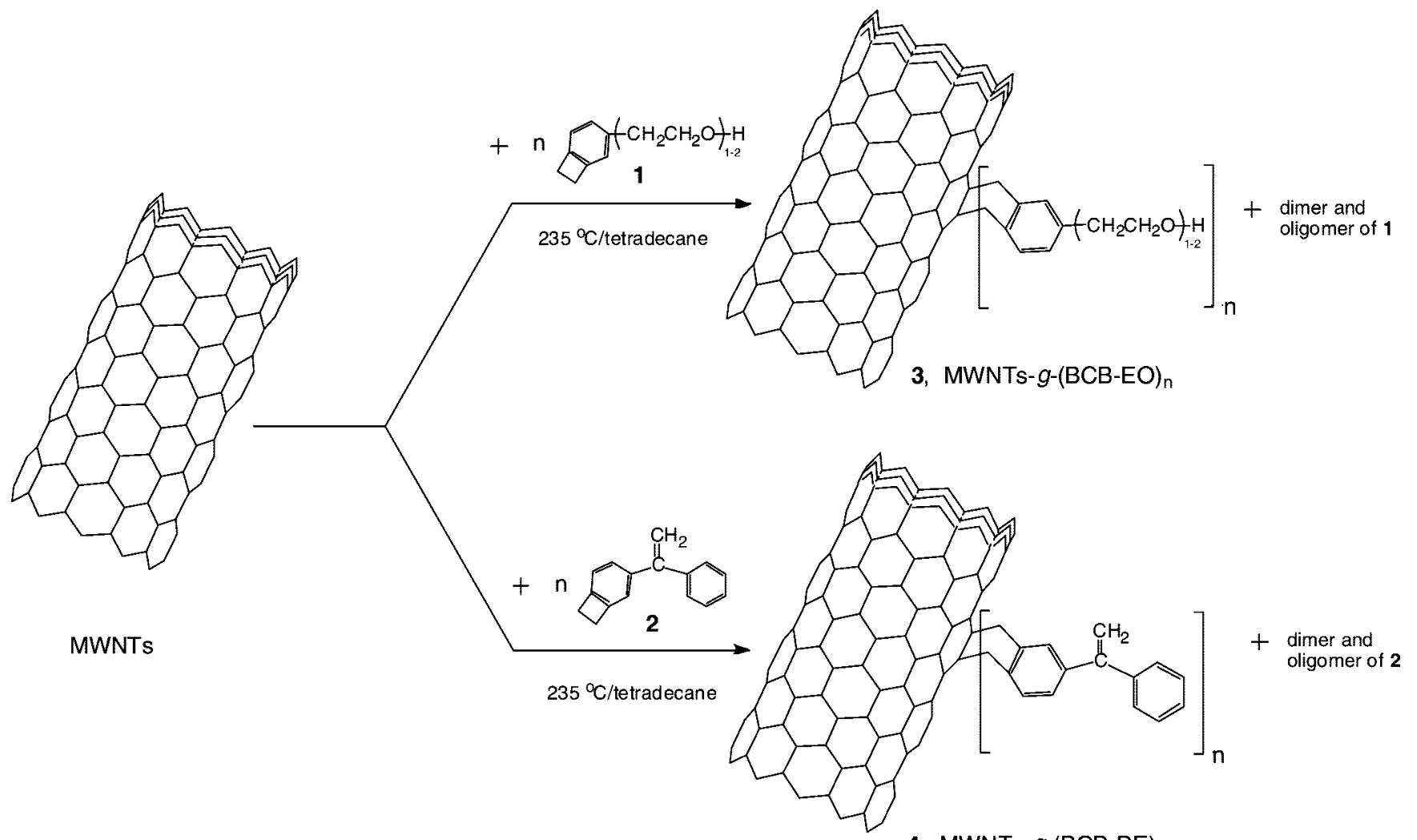

4, MWNTs-g-(BCB-PE)

Table 1. Covalent Functionalization of MWNTs through Diels-Alder Reaction with Benzocyclobutene Derivatives $(1$ and 2$)$ at $230{ }^{\circ} \mathrm{C}$ in Tetradecane

\begin{tabular}{|c|c|c|c|c|c|c|c|c|}
\hline \multirow[b]{2}{*}{ run } & \multirow[b]{2}{*}{$\mathrm{BCB}^{a}$} & \multirow[b]{2}{*}{$\begin{array}{c}{[\mathrm{BCB}]^{b}} \\
\left(\times 10^{4} \mathrm{~mol}\right) \\
\end{array}$} & \multirow[b]{2}{*}{$\begin{array}{c}{[\mathrm{C}]_{\mathrm{MWNT}^{c}}} \\
\left(\times 10^{4} \mathrm{~mol}\right) \\
\end{array}$} & \multirow[b]{2}{*}{$t(\min )$} & \multicolumn{4}{|c|}{ MWNTs- $g$-(BCB-derivative) ${ }_{n}$} \\
\hline & & & & & $\mathrm{BCB}^{d}$ wt $\%$ & $\mathrm{BCB}^{e} \mathrm{~mol} \%$ & $\begin{array}{c}T_{\mathrm{d}-\max -\mathrm{BCB}}{ }^{f} \\
\left({ }^{\circ} \mathrm{C}\right)\end{array}$ & $\begin{array}{c}T_{\mathrm{d}-\max -\mathrm{MWNTs}}{ }^{g} \\
\left({ }^{\circ} \mathrm{C}\right)\end{array}$ \\
\hline 1 & 1-EO & 60.8 & 83.3 & 60 & 23.0 & 1.67 & 395 & 578 \\
\hline 2 & 1-EO & 81.1 & 83.3 & 90 & 47.6 & 3.45 & 391 & 565 \\
\hline 3 & 2-PE & 58.2 & 83.3 & 60 & 20.0 & 1.16 & 470 & 575 \\
\hline 4 & 2-PE & 72.8 & 83.3 & 100 & 54.0 & 3.14 & 472 & 570 \\
\hline
\end{tabular}

${ }^{a}$ Benzocylobutene derivatives, 1 and 2. ${ }^{b}$ Concentration of BCB derivatives; average mol wt of BCB-EO monomeric (60\%) and dimeric EO (40\%) fragment $=165.6 \mathrm{~g} / \mathrm{mol}$, and mol wt of BCB-DPE $=206 \mathrm{~g} / \mathrm{mol} .{ }^{c}[\mathrm{C}]_{\mathrm{MWNT}}=$ grams of MWNTs $/ 12 .{ }^{d}$ Determined using thermogravimetry analysis. ${ }^{e}$ Mole $\%$ with respect to carbon moles $=($ wt $\% \mathrm{BCB}$ in MWNT/mol wt of BCB fragment $) \times 12 .{ }^{f} T_{\mathrm{d}-\text { max-BCB }}=$ maximum decomposition of BCB derivatives in between $200-480{ }^{\circ} \mathrm{C} .{ }^{g} T_{\mathrm{d}-\text { max-MWNTs }}=$ maximum decomposition of MWNTs $\left(480-650{ }^{\circ} \mathrm{C}\right)$.

pale green color developed upon reaction with hydroxyl groups of $\mathbf{3}$ in a heterogeneous solution. After a while, another drop of fresh TMP anions was added to convert the remaining hydroxyl groups into alkoxide anions. It was interesting to notice the development of pale green color during the deprotonation, even though alkoxide anions are known to be colorless. The pale green color of the stirred heterogeneous solution could be attributed to intermolecular interaction of surface alkoxide anions with MWNTs. A slow dropwise titration of hydroxyl groups was continued for about 10 times. At this time the disappearance of the red color became very slow (over $2 \mathrm{~h}$ ), indicating the conversion of a majority of the hydroxyl groups into alkoxide anions. The intensity of the green color also increased at the end of titration, confirming the higher concentration of alkoxide anions generated on the surface of MWNTs. The titration was stopped and the polymerization of EO was started by addition of EO into the heterogeneous solution of MWNTs bearing surface alkoxide initiators in THF.
The polymerization of EO was conducted for different durations $(0.66-48 \mathrm{~h})$, and the results of surface initiated anionic polymerization of EO from 3 are given in Table 2. Samples of poly(ethylene oxide) (PEO) grafted MWNTs (5, MWNT- $g$-(BCB-PEO $)_{n}$ ) were recovered through dissolution in excess THF and filtration using a $0.4 \mu \mathrm{M}$ Teflon membrane. However, for the polymerization carried out for longer durations $(22-48 \mathrm{~h})$, the solution became very viscous and PEO grafted MWNTs (5) could not be filtered through a $0.4 \mu \mathrm{m}$ Teflon membrane, indicating formation of high molecular weight PEO brushes on the surface of MWNTs. The entire solution was precipitated in $n$-hexane to recover MWNTs- $g$-(BCB-PEO) $)_{n}$ as a PEO nanocomposite containing a high percentage of PEO ( $>90$ wt $\%$ ). For low conversion of EO, $\mathbf{5}$ could be separated from the reaction mixture by a simple filtration as described above (Table 2, run 1-4). The recovered quantities of $\mathbf{5}$ were always higher than 100\% (on the basis of $\mathbf{3}$ ) indicating the presence of surface grown PEO. 


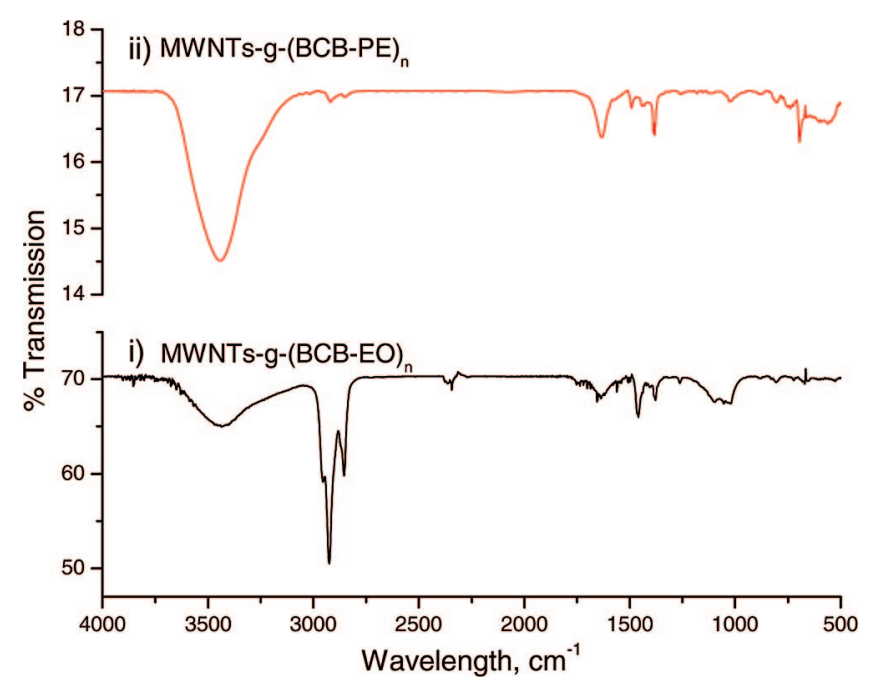

Figure 3. FT-IR spectra of precursor initiator functionalized MWNTs. (i) MWNT- $g$-(BCB-EO) ${ }_{n}$ and (ii) MWNTs- $g$-(BCB-PE) (Table 1, runs 1 and 4).

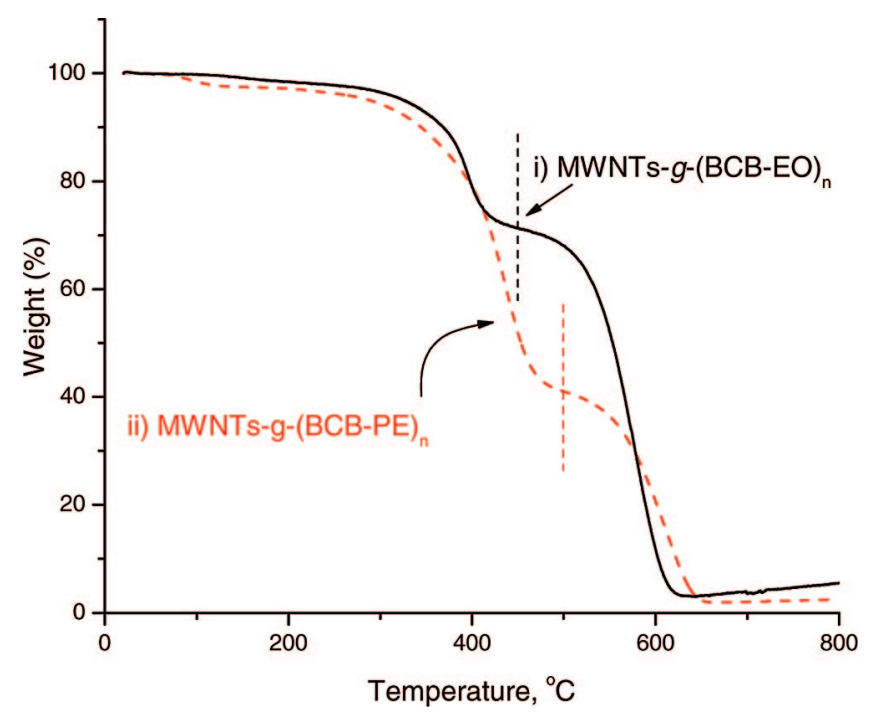

Figure 4. TGA of precursor initiator moieties attached MWNTs in air, (i) MWNT- $g$-(BCB-EO) $)_{n}, 23$ wt \% loss in air between 250 and $450{ }^{\circ} \mathrm{C}$ and (ii) MWNTs- $g$-(BCB-PE) $)_{n} 54$ wt $\%$ loss in air between 250 and $500{ }^{\circ} \mathrm{C}$ (Table 1 , runs 1 and 4 ).

The amount of PEO grown from the surface of MWNTs containing 23 wt $\%$ initiators was determined using TGA. Figure 5 shows thermograms of annealed precursor MWNTs, MWNTs- $g$-(BCB-EO) ${ }_{n}$, and several MWNTs-g-(BCB-PEO) ${ }_{n}$ samples. The polymerization of EO proceeded in a heterogeneous medium and the viscosity of the solution increased with increasing reaction time. At high conversion of EO $(>35 \%)$, the reaction medium became very viscous and solidified. The reaction was terminated by addition of a small amount of methanol and the reaction mixture was diluted with THF and the PEO grafted MWNTs were recovered by precipitation in excess hexane. The surface initiated polymerization of EO carried out for 45 min produced $35.8 \mathrm{wt} \%$ PEO corresponding to $7 \%$ of EO conversion. The TGA of several samples of $\mathbf{5}$ under $\mathrm{N}_{2}$ for different reaction time showed a distinct weight lose at $400{ }^{\circ} \mathrm{C}$ corresponding to the decomposition of PEO from the surface of MWNTs (Figure 5). As CNTs are stable up to $1000{ }^{\circ} \mathrm{C}$ under inert atmosphere, the TGA measurement performed up to $700{ }^{\circ} \mathrm{C}$ under nitrogen for MWNTs- $g$-(BCB-PEO $)_{n}$ showed a single decomposition corresponding to PEO. The maximum decomposition temperature of the surface grown $\mathrm{PEO}$ is slightly higher $\left(\Delta T_{\mathrm{d}-\max } \approx 10^{\circ} \mathrm{C}\right)$ as compared to neat PEO because of the presence of MWNTs. The TGA analysis showed that the growth of PEO increased gradually with increasing reaction time. The reaction carried out for $1.25,2,4$, and $6 \mathrm{~h}$ produced MWNTs- $g$-(BCB-PEO) ${ }_{n}$ samples with 41.4, 45.4, 90.5, and 97.1 wt \% PEO, respectively.

As MWNTs- $g$-(BCB-EO) $)_{n}(23 \mathrm{wt} \%$ ) was used for all the polymerizations with approximately similar initiator concentration, the increase in PEO \% on the surface of MWNTs is attributed to the increase in chain-length of PEO grown from living anionic ring-opening polymerization. The percentage of EO conversion as determined from the recovered yield of $\mathbf{5}$ from various reactions also increases with increasing reaction time, supporting the living nature of surface initiated ring-opening polymerization (Table 2, run $1-4)$. The results confirm that through living anionic ringopening polymerization, it is possible to obtain MWNTs- $g$ $(\mathrm{BCB}-\mathrm{PEO})_{n}$ nanocomposites with very long PEO brushes. Enhanced growth of PEO from the surface of MWNTs produced PEO nanocomposites with as low as $2 \mathrm{wt} \%$ MWNTs.

The FT-IR spectrum of $\mathbf{5}$ exhibits two major stretching frequencies centered at 2880 and $1110 \mathrm{~cm}^{-1}$ indicating the presence of $-\mathrm{CHs}$ and $\mathrm{C}-\mathrm{O}$ of $\mathrm{PEO}$, respectively (Figure 6a). The structural integrity of the surface grafted MWNTs was confirmed by Raman spectroscopy, which showed both the disorder (d) and tangential (t) bands at 1334 and 1590 $\mathrm{cm}^{-1}$ respectively. An increase in the peak ratio of $I_{\mathrm{d}} / I_{\mathrm{t}}$ in $\mathbf{5}$ as compared to the precursor initiator attached MWNTs is attributed to enhanced attachment of PEO chains (Figure 6b). The ${ }^{1} \mathrm{H}$ NMR of 5 showed signals corresponding to methylene protons of PEO at $3.61 \mathrm{ppm}$ (see the Supporting Information).

The initiation and the propagation processes in SIP of EO from the MWNTs were slow as indicated from the monomer conversion for $48 \mathrm{~h}(<57 \%)$. This could be attributed to the heterogeneous nature of the polymerization. It appears that the rate of polymerization is also influenced by the efficiency in which the reagents are mixed in a heterogeneous SIP. It was noticed that the development of viscosity in the reaction medium was faster when the reaction was conducted under rapid stirring. On the other hand, a significantly slower polymerization was observed when the reaction was carried out without stirring, and no viscosity increase was observed under these conditions. The recovered MWNTs- $g$-(BCB$\mathrm{PEO})_{n}$ was gray in color despite the fact that it contains a very high percentage of PEO grafts $(<98 \mathrm{wt} \%)$.

A control experiment was also carried out under identical conditions in the presence of pristine MWNTs bearing no functional groups in THF. In this case, the addition of the first drop of triphenylmethyl potassium into THF containing MWNTs produced a red coloration. The red color of the anion persisted over a $12 \mathrm{~h}$ period in THF at room temperature. Upon addition of EO, the red color of the anion disappeared immediately and the solution turned pale green. The polymerization was allowed to proceed at $50{ }^{\circ} \mathrm{C}$ for $20 \mathrm{~h}$. 
Scheme 3. Surface-Initiated Anionic Polymerization of Ethylene Oxide from MWNTs- $g$-(BCB-EO) $)_{n}$ Surface

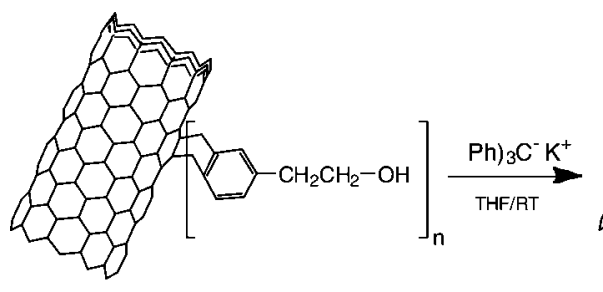

3, MWNTs-g-(BCB-EO)
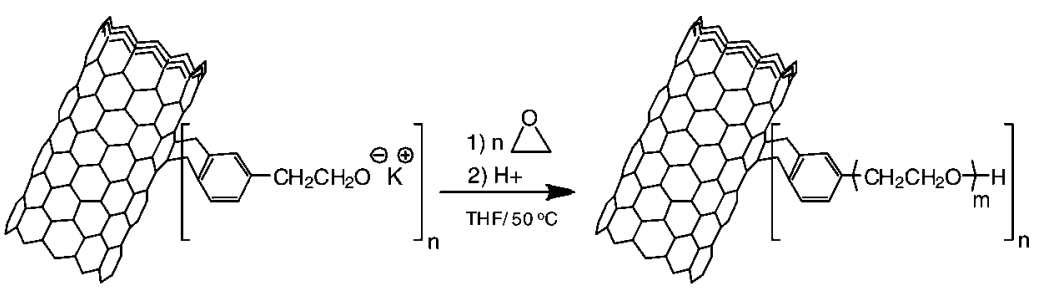

5. MWNTs-g-(BCB-PEO)

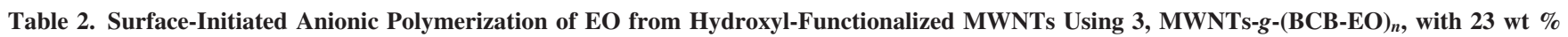
Precursor Initiator in the Presence of Potassium Counterion in THF at $25{ }^{\circ} \mathrm{C}$

\begin{tabular}{|c|c|c|c|c|c|c|c|c|c|}
\hline \multirow[b]{2}{*}{ run } & \multirow[b]{2}{*}{$\begin{array}{c}{[\mathrm{C}]_{\mathrm{MWNT}^{a}}{ }^{2}} \\
\left(\times 10^{4} \mathrm{~mol}\right)\end{array}$} & \multirow[b]{2}{*}{$\begin{array}{c}{[\mathrm{I}]_{\mathrm{MWNT}^{b}}} \\
\left(\times 10^{5} \mathrm{~mol}\right)\end{array}$} & \multirow[b]{2}{*}[\mathrm{EO}]{$(\mathrm{mol} / \mathrm{L})$} & \multirow[b]{2}{*}{$t(\mathrm{~h})$} & \multirow[b]{2}{*}{$\begin{array}{c}\text { conv. }^{c} \\
{[\mathrm{EO}](\%)}\end{array}$} & \multicolumn{4}{|c|}{ MWNTs- $g$-(BCB-PEO $)_{\mathrm{n}}$} \\
\hline & & & & & & $\mathrm{PEO}^{d}(\%)$ & $\mathrm{MWNTs}^{e} \%$ & $\begin{array}{c}M_{\mathrm{n}, \mathrm{app}} \times 10^{-3 f} \\
(\mathrm{~g} / \mathrm{mol})\end{array}$ & $M_{\mathrm{w}} / M_{\mathrm{n}}$ \\
\hline 1 & 6.66 & 1.11 & 6.29 & 0.66 & 7.0 & 35.8 & 64.2 & & \\
\hline 3 & 6.66 & 1.11 & 6.29 & 2 & 10.0 & 45.4 & 54.6 & & \\
\hline 4 & 6.66 & 1.11 & 6.29 & 4 & 18.0 & 90.5 & 9.5 & 104.0 & 1.33 \\
\hline 5 & 5.83 & 0.97 & 8.38 & 6 & 22.0 & 97.1 & 2.9 & 144.0 & 1.78 \\
\hline
\end{tabular}

${ }^{a}$ Moles of carbon in MWNTs. ${ }^{b}$ Moles of $I=(($ wt $\% \mathrm{I} / \mathrm{mol}$ wt of I fragment $) / 100) \times \mathrm{g}$ of $\mathbf{3}$ taken for the reaction. ${ }^{c}$ Conversion of EO determined by gravimetry. ${ }^{d}$ Weight percent PEO on the surface of MWNTs determined from TGA. ${ }^{e}$ Determined by TGA including residual metal oxide present in the MWNTs. ${ }^{f}$ Apparent number average molecular weight of MWNTs- $g$-(BCB-PEO) $n$ corresponding to PS calibration from size exclusion chromatography.

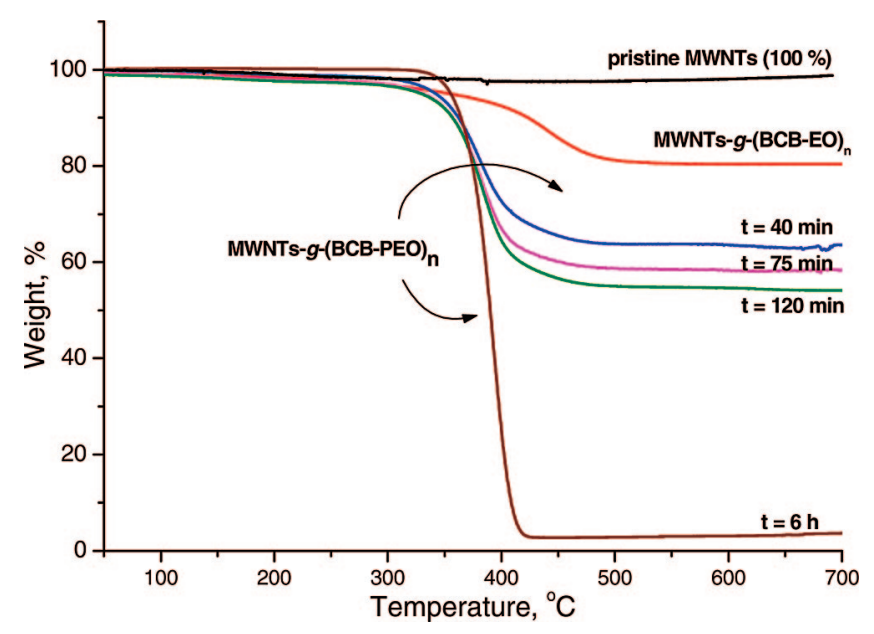

Figure 5. TGA of MWNTs- $g-(\mathrm{BCB}-\mathrm{PEO})_{n}(\mathbf{5})$ at various reaction times in the surface-initiated anionic polymerization of ethylene oxide from MWNTs$g-(\mathrm{BCB}-\mathrm{EO})_{n}(\mathbf{3})$ in $\mathrm{THF}$ at $40{ }^{\circ} \mathrm{C}$.

The viscosity of polymerization solution increased gradually, and the reaction was terminated with a small amount of methanol. The entire solution was diluted with excess THF and filtered using a $0.4 \mu \mathrm{m}$ Teflon membrane. Unlike SIP reactions, the diluted polymerization solution could be filtered freely and the MWNTs were recovered quantitatively. The TGA analysis of the recovered MWNTs showed an insignificant weight loss at temperatures between $250-450{ }^{\circ} \mathrm{C}$, indicating the absence of PEO on the surface of MWNTs. The results of this control experiment suggest that the PEO growth from the covalently modified MWNTs is only from the hydroxyl groups of MWNTs- $g$-(BCB-EO) .

The functionalized MWNTs, 3 with 23 wt $\%$ of the ethylene oxide moiety are soluble in water at concentrations up to $4 \mathrm{mg} / \mathrm{mL}$. Similarly, all the samples of PEO grafted MWNTs are soluble in water, THF, and $\mathrm{CHCl}_{3}$ (see the
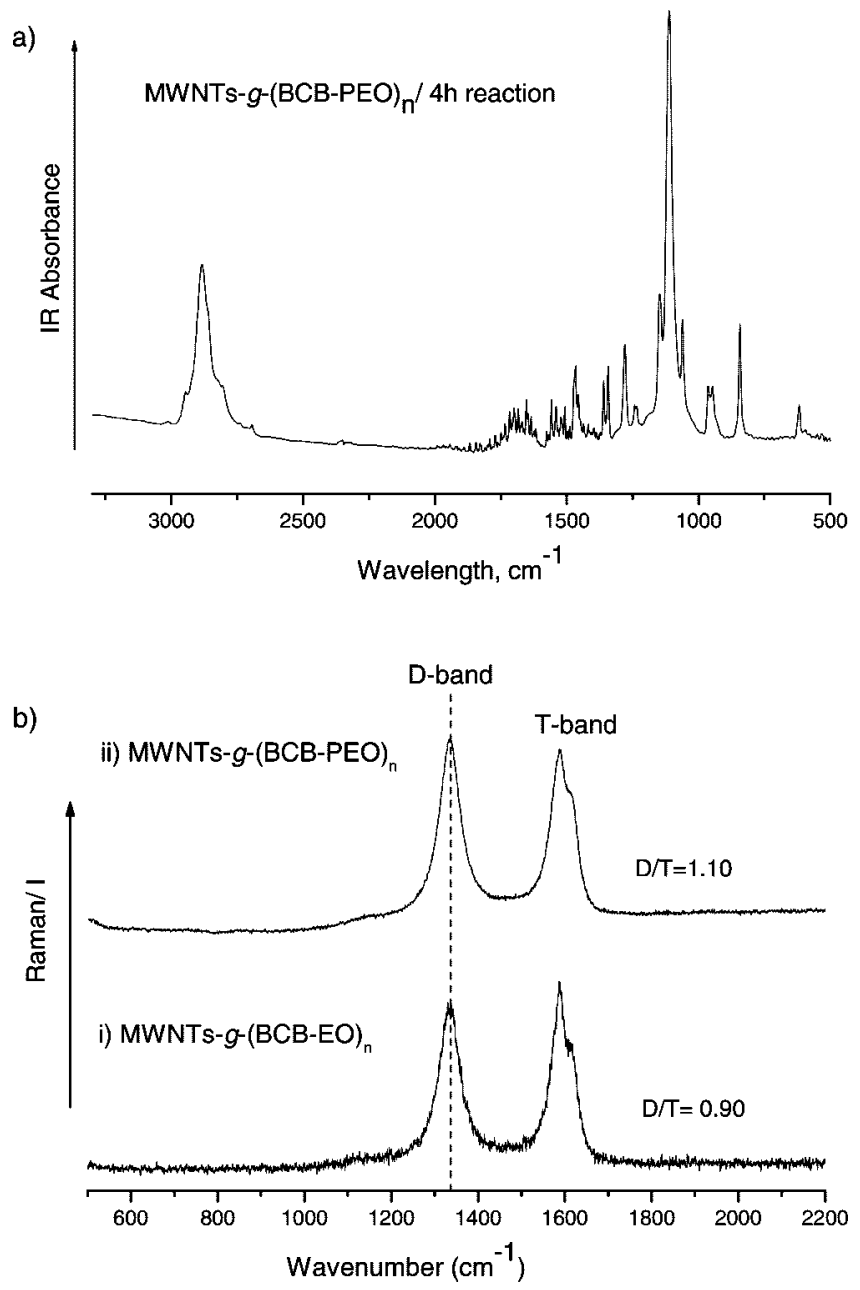

Figure 6. (a) FT-IR of MWNTs- $g$-(BCB-PEO) ${ }_{n}$ recovered from the reaction carried out for $4 \mathrm{~h}$ in THF at $50{ }^{\circ} \mathrm{C}$ and b) Raman spectra of precursor initiator (3) and surface-initiated PEO (5) attached MWNTs, (i) MWNTs$g$-(BCB-EO $)_{n}$ and (ii) MWNTs- $g$-(BCB-PEO) $n$. 

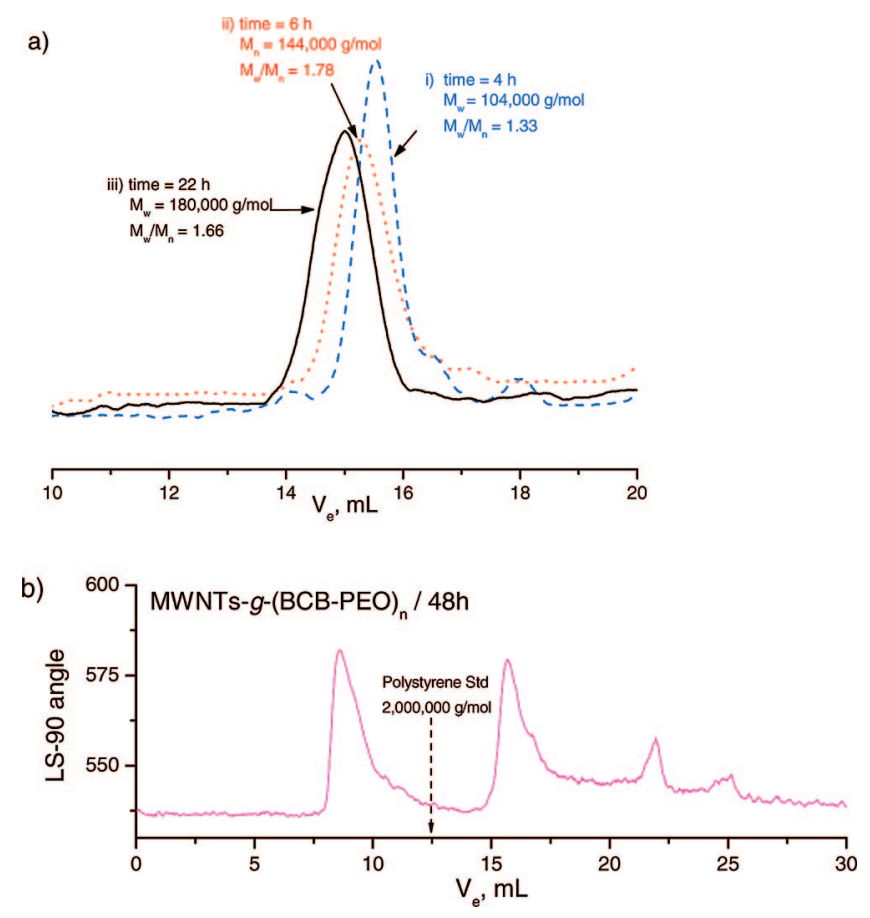

Figure 7. SEC traces of (a) MWNTs- $g$-(BCB-PEO) $n$ at different reaction times indicating the a gradual growth of $\mathrm{PEO}$, (i) 90.5, (ii) 97.1, and (iii) 98.0 wt \% PEO; and (b) light scattering detector for MWNTs- $g$-(BCBPEO $)_{n}$ with 95.9 wt \% PEO exhibiting presence of low concentration of high- and low-molecular-weight products (run 7, Table 2).

Supporting Information). The aqueous solution of $\mathbf{5}$ could be analyzed for molecular weight determination of PEO grafted MWNT brushes. It should be mentioned that the multiple size distributions of pristine MWNTs with regard to their length, diameter, and number of layers, together with the heterogeneous nature of the polymerization, produce products that differ in size and mass and hence the samples of PEO grown MWNTs using anionic polymerization are not expected to have a narrow molecular weight distribution. This appears to be the fact as the SEC traces of $\mathbf{5}$ at different reaction times showed broad apparent molecular weight distributions $\left(M_{\mathrm{w}} / M_{\mathrm{n}}>1.1, M_{\mathrm{w}}\right.$ and $M_{\mathrm{n}}$ are the weight and number average molecular weights, respectively) (Table 2, Figure 7a). The actual molecular weight of hairy PEO on the surface of MWNTs would be difficult to determine because of the broad distribution of MWNTs as well as the statistical distribution of initiator groups.

The SEC analysis of these cylindrical brushes is difficult due to their dimensions. It is expected that most of them would pass through interparticle volume rather than pores of the SEC column material. Although, CNTs have been purified previously using SEC, molecular separation was not achieved. ${ }^{45,46}$ The experimentally obtained molecular weight of $\mathbf{5}$ is only apparent as it consists of the volume occupied by the MWNT with the number of grafted chains on it. Moreover, a broad distribution of MWNTs with respect to length and diameter also adds to the complexity in correlating

(45) Zhao, B.; Hu, H.; Niyogi, S.; Itkis, M. E.; Hamon, M. A.; Bhowmik, P.; Meier, M. S.; Haddon, R. C. J. Am. Chem. Soc. 2001, 123, 1167311677.

(46) Niyogi, S.; Hu, H.; Hamon, M. A.; Bhowmik, P.; Zhao, B.; Rozenzhak, S. M.; Chen, J.; Itkis, M. E.; Meier, M. S.; Haddon, R. C. J. Am. Chem. Soc. 2001, 123, 733-734.

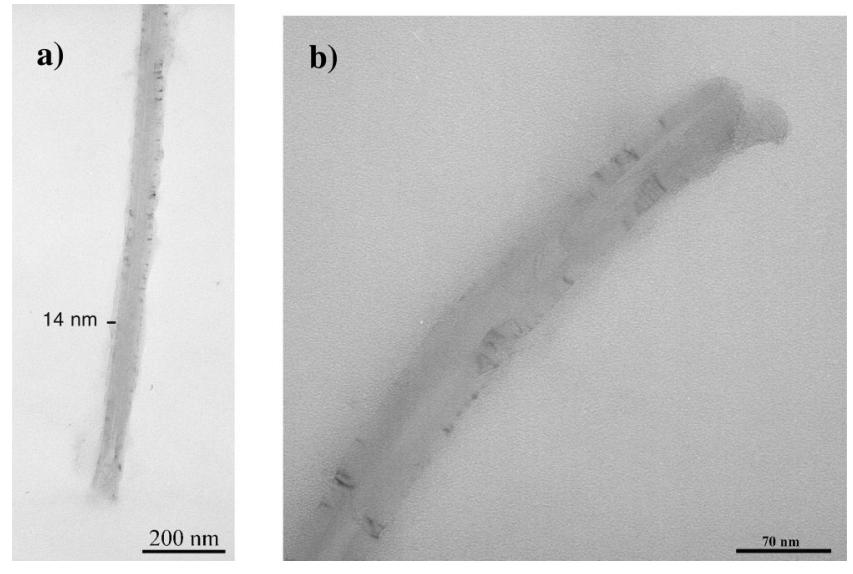

Figure 8. TEM images of MWNTs- $g$-(BCB-PEO) ${ }_{n}(\mathrm{a}, \mathrm{b})$ with nonuniform PEO grafted chain on the surface of MWNTs (Table 2, run 1).

initiator concentration with respect to apparent molecular weight. The grafted chains are covalently attached to $\mathrm{sp}^{2}$ carbons of the MWNTs through carbon-carbon bond and hence are not cleavable for characterization. The apparent molecular weights of the polymer grafted tubes obtained by SEC analysis in relation to polystyrene standards should be used for comparison purpose only. Interestingly, the SEC elution profile of $\mathbf{5}$ showed a gradual shift to lower elusion volume with respect to the reaction time suggesting that the overall apparent number-average molecular weight of MWNTs$g$-(BCB-PEO $)_{n}$ brushes increases with increasing reaction time (Figure 7a). In some cases, a small fraction of PEO grafted MWNTs exhibited very high molecular weight, which could be detected only by the light scattering detector (Figure 7b). It was not possible to identify the presence of multiple species in conventional SEC using concentration detector (refractive index detector, RI), as they are present in low concentration. Nonetheless, the light scattering detector confirmed the presence of low concentrations of multiple species of PEO grafted MWNTs exhibiting larger and smaller dimensions.

The PEO-grafted MWNTs samples were characterized using TEM. Accordingly, a dilute solution of $\mathbf{5}$ with 35.8 wt \% PEO in $\mathrm{CHCl}_{3}$ was drop-coated on to a carbon grid and viewed through TEM. A substantial amount of amorphous organic layer up to $\sim 14 \mathrm{~nm}$ thickness on the surface of MWNTs- $g$-(BCB-PEO) ${ }_{n}$ was identified in the TEM images (Figure 8a). A large portion of MWNTs is covered with the grafted PEO and some portion of MWNTs could not be visible clearly in the TEM images indicating extensive coverage of polymer around the tubes (Figure 8b). The surface of MWNTs is covered with the PEO layer inhomogeneously, which suggests a nonuniform grafting of PEO chains around the surface of carbon nanotubes.

Surface-Initiated Anionic Polymerization of Styrene from MWNTs- $g$-(BCB-PE) $)_{n}$. Ajayan and co-workers have attempted recently to generate carbanion on the surface of SWNTs using a direct addition of sec-BuLi to their $\mathrm{sp}^{2}$ carbon network. ${ }^{38}$ The polymerization of styrene was conducted in situ in the presence of surface anions and free $s e c-\mathrm{BuLi}$ to produce a polystyrene nanocomposite. However, a careful analysis of the nanocomposite indicated that the initiation of styrene from SWNTs-anions was inefficient with 
Scheme 4. Surface Initiated Anionic Polymerization of Styrene Using MWNTs- $g$-(BCB-PE) ${ }_{n}$ in Benzene

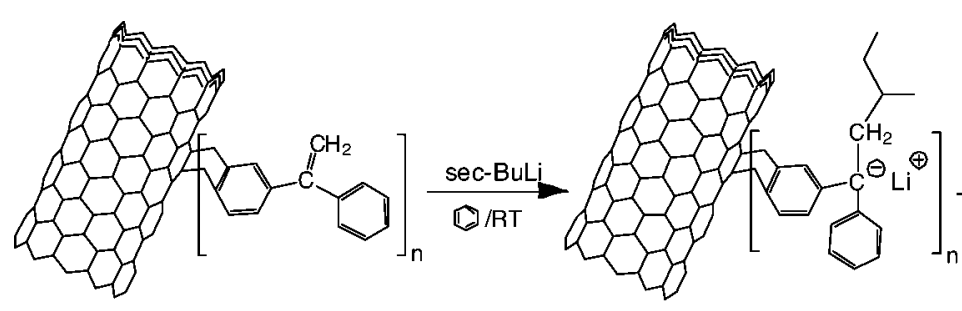

4, MWNTs-g-(BCB-PE)

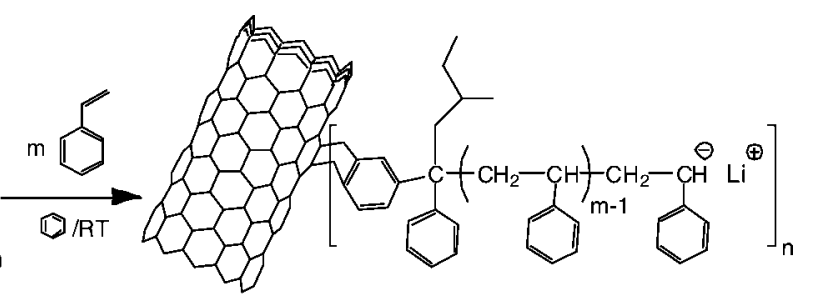

6, MWNTs- $g$-(BCB-PS)

Table 3. Surface-Initiated Anionic Homopolymerization and Block Copolymerization of Styrene and Isoprene [M] from 1-Benzocyclobutene-1'-phenylethylene-Functionalized MWNTs Using 4, MWNTs- $g$-(BCB-PE) ${ }_{n}$ with 54.0 wt \% precursor initiator in Benzene at $25^{\circ} \mathrm{C}$

\begin{tabular}{|c|c|c|c|c|c|c|c|c|c|c|}
\hline \multirow[b]{2}{*}{ Run } & \multirow[b]{2}{*}{$\begin{array}{c}{[\mathrm{C}]_{\mathrm{MWNT}^{a}}} \\
\left(\times 10^{4} \mathrm{~mol}\right)\end{array}$} & \multirow[b]{2}{*}{$\begin{array}{c}{[\mathrm{I}]_{\mathrm{MWNT}^{b}}} \\
\left(\times 10^{5} \mathrm{~mol}\right) \\
\end{array}$} & \multirow[b]{2}{*}[\mathrm{M}]{$(\mathrm{mol} / \mathrm{L})$} & \multirow[b]{2}{*}{$t(\mathrm{~h})$} & \multirow[b]{2}{*}{$\begin{array}{l}\text { Conv. }^{c} \\
{[\mathrm{M}](\%)}\end{array}$} & \multicolumn{5}{|c|}{ MWNTs- $g$-(BCB-PS $)_{\mathrm{n}}$} \\
\hline & & & & & & $\operatorname{PS}^{d}(\%)$ & $T_{\mathrm{d}-\max } \mathrm{PS}\left({ }^{\circ} \mathrm{C}\right)$ & $\operatorname{MWNTs}^{e}(\%)$ & $\begin{array}{c}\mathrm{M}_{\mathrm{n}, \mathrm{app}}^{f} \\
\left(\times 10^{-3} \mathrm{~g} / \mathrm{mol}\right)\end{array}$ & $M_{\mathrm{w}} / M_{\mathrm{n}}$ \\
\hline \multicolumn{11}{|c|}{ Monomer, $[\mathrm{M}]$ : Styrene } \\
\hline 2 & 17.5 & 5.50 & 3.85 & 1.0 & 18.0 & 92.0 & 350 & 8.0 & 655.0 & 1.51 \\
\hline 3 & 12.5 & 3.93 & 0.86 & 1.50 & 30.0 & 86.2 & 397 & 13.8 & 422.0 & 1.49 \\
\hline 4 & 12.5 & 3.93 & 0.86 & 1.50 & 33.0 & 90.6 & 393 & 9.4 & 378.0 & 1.48 \\
\hline & & & $0.74^{g}$ & 18 & 100 & 99.0 & & $<1.0$ & 305.0 & 1.36 \\
\hline
\end{tabular}

${ }^{a}$ Moles of carbon in MWNTs. ${ }^{b}$ Moles of $I=(($ wt $\% \mathrm{I} / \mathrm{mol} \mathrm{wt}$ of I fragment $) / 100) \times \mathrm{g}$ of $\mathbf{4}$ taken for the reaction. ${ }^{c}$ Conversion of PS determined by gravimetry. ${ }^{d}$ Weight percent PS on the surface of MWNTs determined from TGA. ${ }^{e}$ Determined by TGA including residual catalyst in the MWNTs. ${ }^{f}$ Apparent number-average mole cular weight of MWNTs- $g$-(BCB-PS) $)_{n}$ corresponding to PS calibration from size exclusion chromatography. ${ }^{g}$ Isoprene addition to the living chain-ends of PS.

only $15-20$ wt \% polystyrene grafted from the surface. We independently studied grafting reactions of alkyllithium and living polymeric-macroanions directly on to the $\mathrm{sp}^{2}$ carbons of MWNTs. ${ }^{15}$ We confirmed that the amount of sec-BuLi addition is inefficient and limited to less than $0.1 \mathrm{~mol} \%{ }^{15}$ To have an efficient surface initiation of styrene, we decided to generate carbanion sites away from the $\mathrm{sp}^{2}$ network of carbon nanotubes.

Anionic polymerization of styrene is typically performed using sec-BuLi as initiator in a hydrocarbon medium. ${ }^{41}$ Quirk et al. and others have shown that a delocalized anion such as an adduct of 1,1'-diphenylethylene with sec-BuLi (diphenylhexyl lithium) can also be used to initiate styrene polymerization. ${ }^{43}$ Our strategy to initiate styrene and diene homo and block copolymers from the surfaces of MWNTs is to generate such a delocalized carbanion through addition of sec-BuLi with 4 (MWNTs-g-(BCB-PE) $)_{n}$ ), which has 1-benzocyclobutene-1'-phenylethylene (2) covalently attached to the surface of MWNTs (Scheme 4).

The MWNTs with 54 wt \% 1-benzocyclobutene-1'phenylethylene (2) were first placed in a specially designed reactor as described in the Experimental Section. A known quantity of $\mathbf{4}$ was reacted with excess sec-BuLi in benzene under a vacuum for $12 \mathrm{~h}$ at room temperature. It was difficult to ascertain the color of the generated carbanion on the surface. After stirring for $12 \mathrm{~h}$, the MWNTs were allowed to settle to the bottom of the flask and the excess sec-BuLi was decanted carefully into an empty ampule. The initiator functionalized MWNTs were then washed thoroughly with freshly distilled benzene several times to remove any adsorbed sec-BuLi. The absence of free sec-BuLi was confirmed through examination of a decanted small amount of benzene solution for styrene initiation. Thorough washing was confirmed by the absence of orange coloration in attempted styrene initiation. The surface-initiated polymerization of styrene was then started by mixing styrene into the reactor containing MWNTs bearing the surface anions. During the polymerization, the color of the reaction medium under fast stirring appeared to be black because of MWNTs and the orange color of the propagating anions could not be discerned. The initiation and propagation of styrene was confirmed as the heterogeneous polymerization solution became gradually viscous and homogeneous over time. Several polymerizations were performed in benzene at room temperature and the results are given in Table 3 .

The reaction was terminated using a small amount of methanol. The polystyrene grafted MWNTs, 6, MWNTs- $g$ $(\mathrm{BCB}-\mathrm{PS})_{n}$, were recovered through precipitation and dried at $60{ }^{\circ} \mathrm{C}$ under vacuum for $4 \mathrm{~h}$. The TGA of 6 for the reaction carried out for 45 min showed $97.8 \%$ weight loss corresponding to polystyrene in the temperature range between 350 and $450{ }^{\circ} \mathrm{C}$. This amount corresponds to a monomer conversion of $15 \%$ on the basis of the yield obtained gravimetrically. However, a small portion of the MWNTs could be seen settled in the reactor during the polymerization as a result of slow initiation. On the other hand, the polymerizations carried out for 90 min (Table 3, runs 4 and 5) showed a complete dissolution of MWNTs during the polymerization. The results suggest that the initiation of styrene from the surface of MWNTs in a heterogeneous medium is very slow and has a long induction period. A slow initiation process from the MWNTs surfaces leads to disproportionate chain-growth in a heterogeneous medium.

A control experiment was carried out in which a small amount of sec-BuLi was left in the solution in the presence of pristine MWNTs. Upon addition of styrene, an orange 


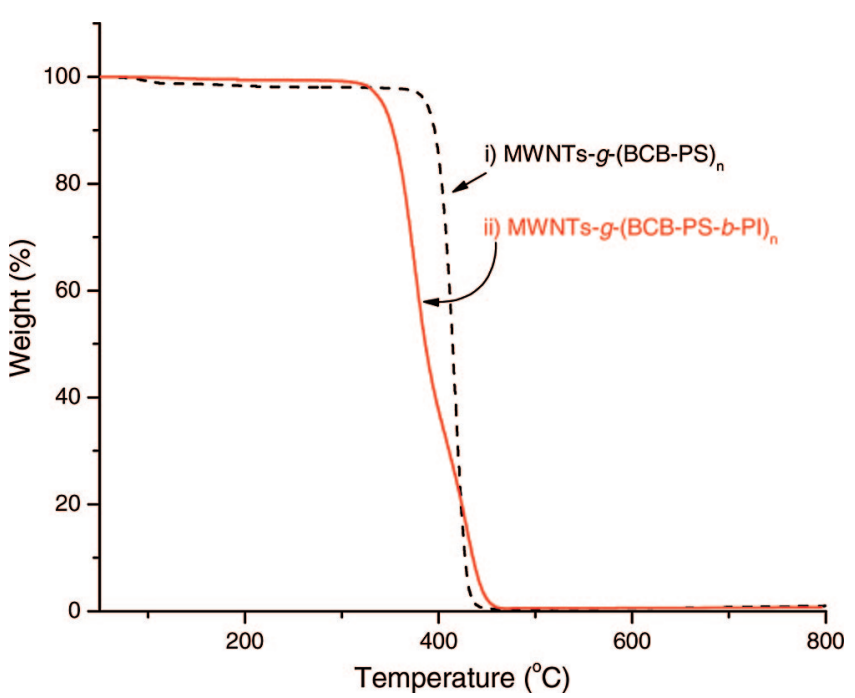

Figure 9. TGA of MWNTs functionalized with (i) first block, MWNTs$g$-(BCB-PS) $)_{n}$, and (ii) diblock, MWNTs- $g$-(BCB-PS- $b$-PI) $n_{n}$ under a $\mathrm{N}_{2}$ atomsphere.

color appeared immediately indicating initiation of styrene from the free initiator present in the solution. The polymerization was terminated after $10 \mathrm{~h}$ and the recovered MWNTs from the reaction mixture showed no decomposition between $250-450{ }^{\circ} \mathrm{C}$ in TGA confirming the absence of PS on the surface. The PS was recovered quantitatively by precipitation in methanol. These results confirm that the polymerization proceeds preferentially in solution and no, or insignificant initiation occurred from the surface. In the case of initiation from the surface, the initiation is sterically hindered leading to a slow initiation. This is in accordance with Adronov and co-workers who observed a drastically different reactivity for SWNTs surface attached radical initiators as compared to sacrificial radical initiators added in surface initiated ATRP. ${ }^{16}$ The heterogeneous nature of the reaction also prevents easy accessibility of initiating sites for the polymerization, leading to an overall slow initiation.

The slow initiation could lead to a broad distribution of polymer chains on the surface. The PS grafts from once initiated sites of MWNTs could grow longer because of a relatively faster propagation of solvated living chain under heterogeneous condition. Nonetheless, in all cases, a very high percentage of PS was present on the MWNTs (86-97 wt $\%$ ) as determined by TGA. The TGA of $\mathbf{6}$ under a nitrogen atmosphere showed a single decomposition at 300 to $450{ }^{\circ} \mathrm{C}$ corresponding to PS $\left(T_{\mathrm{d}-\max }=417{ }^{\circ} \mathrm{C}\right.$ ) (Figure $9 i)$. Anionic surface initiated polymerization gave very high weight percent of PS on the MWNTs as compared to atomtransfer radical surface initiated polymerizations $(<75 \mathrm{wt}$ $\%) .{ }^{47-49}$ Moreover, the living anionic chain ends open up several possibilities of producing tailored di- and triblock copolymer grafts from carbon nanotubes using sequential monomer addition. In spite of the fact that a very low

(47) Kong, H.; Gao, C.; Yan, D. Macromolecules 2004, 37, 4022-4030.

(48) Qin, S.; Qin, D.; Ford, W. T.; Resasco, D. E.; Herrera, J. E. Macromolecules 2004, 37, 752.

(49) Fragneaud, B.; Masenelli-Varlot, K.; Gonzalez-Montiel, A.; Terrones, M.; Cavaille, J.-V. Chem. Phys. Lett. 2006, 419, 567-573.
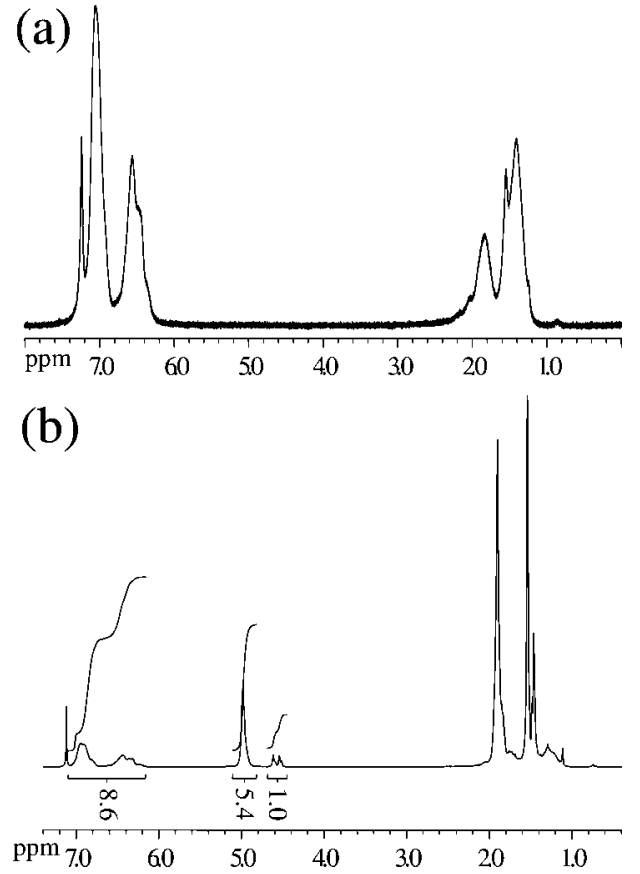

Figure 10. ${ }^{1} \mathrm{H}$ NMR of (i) MWNTs- $g$-(BCB-PS) $n$ and (ii) MWNTs- $g$-(BCBPS- $b$-PI $)_{n}$ in $\mathrm{CDCl}_{3}$ showing signals corresponding to PS and PS- $b$-PI.

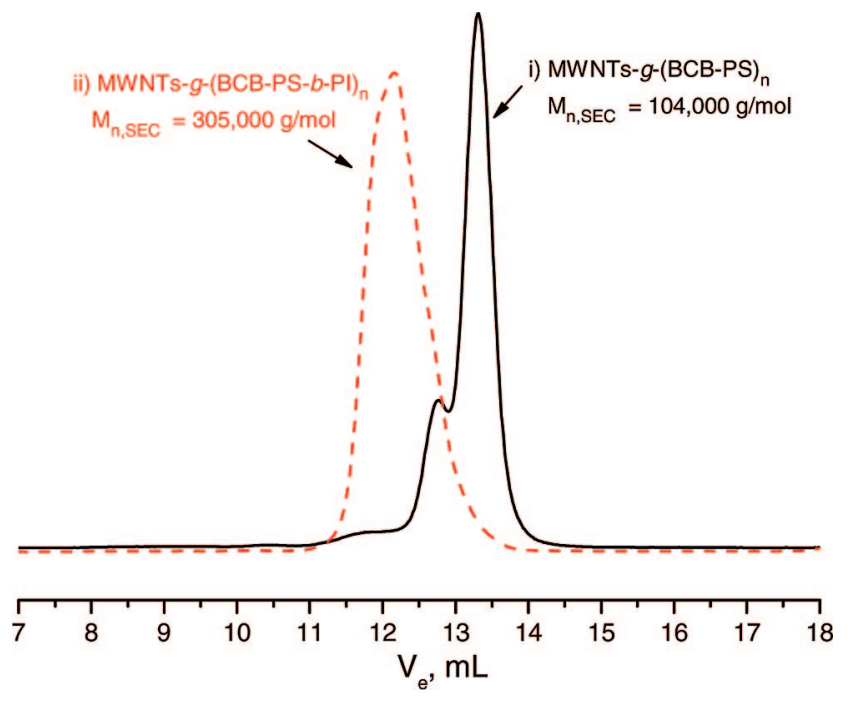

Figure 11. SEC chromatograms of PS and PS- $b$-PI copolymers grown from the surface of MWNTs though surface-initiated anionic polymerization: (i) MWNTs- $g$-(BCB-PS $)_{n}$ containing higher-molecular-weight fractions, $M_{\mathrm{n}, \mathrm{SEC}}$ $=104000 \mathrm{~g} / \mathrm{mol} ; M_{\mathrm{w}} / M_{\mathrm{n}}=1.26$, and (ii) MWNTs- $g$-(BCB-PS- $b$-PI) ${ }_{n}$, $\mathrm{M}_{\mathrm{n}, \mathrm{SEC}}=305000 \mathrm{~g} / \mathrm{mol} ; M_{\mathrm{w}} / M_{n}=1.36$.

percentage of MWNTs is present in $\mathbf{6}$, the thermal stability of the grafted PS was found to be $10{ }^{\circ} \mathrm{C}$ higher than the bulk PS. It is also important to note that the determination of the actual quantity of MWNTs in $\mathbf{6}$ was complicated because of the presence of a small second decomposition of PS at $T_{\text {d-max }} \sim 530{ }^{\circ} \mathrm{C}$ in air which merges with the decomposition of MWNTs $\left(T_{\mathrm{d}-\max } \sim 580^{\circ} \mathrm{C}\right)$. A large amount of residual weight, in some cases, could be attributed to the presence of small pieces of glass present from fracturing break-seals in transfers in the all glass reactors. Thus, we believe that the amount of MWNTs present in MWNTs- $g$ $(\text { BCB-PS })_{n}$ should be treated as an upper limit and actual amount is lower than listed in Table 3. 

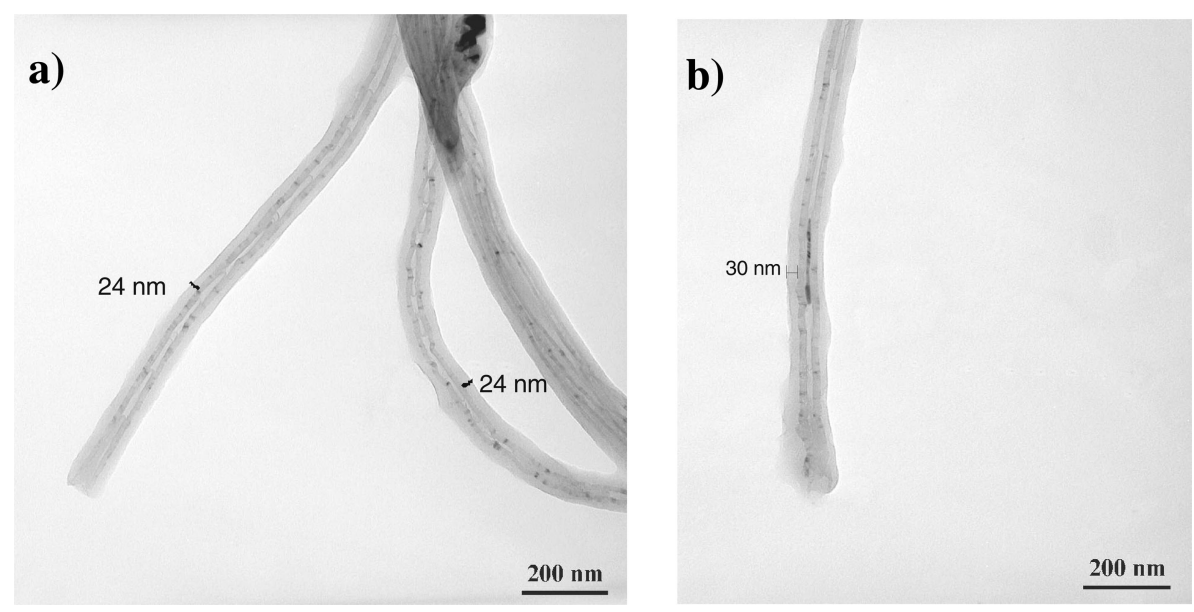

Figure 12. (a, b) TEM of surface-grown PS from 4 exhibiting up to $30 \mathrm{~nm}$ PS layer on the surface of MWNTs- $g$-(BCB-PS $)_{n}$.

The FT-IR spectroscopy analysis of $\mathbf{6}$ confirmed the presence of vibration frequencies centered at $2924 \mathrm{~cm}^{-1}$ (-CHs), $3063 \mathrm{~cm}^{-1}$ (aromatic) corresponding to PS (see the Supporting Information). Similarly, the Raman spectrum of $\mathbf{6}$ showed the presence of both the disorder and the tangential bands at $\sim 1345 \mathrm{~cm}^{-1}$ and $\sim 1595 \mathrm{~cm}^{-1}$ corresponding to MWNTs (Supporting Information). The samples of $\mathbf{6}$ were all soluble in $\mathrm{CHCl}_{3}$ and THF. An attempt was made to grow a diblock copolymer with isoprene from the surface of MWNTs using sequential monomer addition method (Table 3 , run 5). Accordingly, styrene was first polymerized to $100 \%$ conversion by allowing the surface-initiated polymerization to proceed for $24 \mathrm{~h}$. A small amount of polymerization solution was withdrawn into an ampule for characterization of first block growth and then the isoprene ampule was opened to the solution of living polystyryllithium anions of MWNTs. The aliquot and the diblock copolymerization solutions were homogeneous and black in color indicating the presence of dispersed MWNTs. The diblock copolymerization was continued for $18 \mathrm{~h}$ at room temperature and precipitated into excess methanol. The precipitated MWNTs$g$-(BCB-PS $)_{n}$ and MWNTs- $g$-(BCB-PS- $b$-PI $)_{n}$ were gray in color indicating the presence of MWNTs. The TGA of the MWNTs- $g$-(BCB-PS- $b$-PI) ${ }_{n}$ performed under nitrogen showed $\sim 99$ wt $\%$ decomposition with two weight loses at $T_{\mathrm{d}-\max }=$ $375^{\circ} \mathrm{C}$ and $T_{\mathrm{d}-\max }=431{ }^{\circ} \mathrm{C}$ corresponding to the degradation of PI and PS blocks from the surface, respectively (Figure 9ii).

The ${ }^{1} \mathrm{H}$ NMR of the solutions of MWNTs- $g$-(BCB-PS) and MWNTs- $g$-(BCB-PS- $b-\mathrm{PI})_{n}$ in $\mathrm{CDCl}_{3}$ confirmed the presence of protons corresponding to the surface grown PS and PS- $b$-PI, respectively (Figure 10). The SEC of MWNTs$g$-(BCB-PS $)_{n}$ exhibited a major signal along with high molecular weight shoulders (Figure 11). The apparent number-average molecular weight of the PS brushes along with MWNTs was $M_{\mathrm{n}, \mathrm{SEC}}=104000 \mathrm{~g} / \mathrm{mol}$ with a moderate molecular weight distribution of 1.26. The growth of isoprene from the living chain-ends of MWNTs- $g$-(BCB-PS) $n_{n}$ was evident as the SEC elution profile shifted substantially to lower volume indicating the formation of higher-molecularweight diblock grafts $\left(M_{\mathrm{n}, \mathrm{SEC}}=305000 \mathrm{~g} / \mathrm{mol}\right)$ (Figure 11). Although the molecular weight distribution of MWNTs- $g$ (BCB-PS- $b-\mathrm{PI})_{n}$ was broad $\left(M_{\mathrm{w}} / M_{\mathrm{n}}=1.36\right)$, it did not reveal the presence of the precursor PS grafted MWNTs. This suggests that all the active PS chain-ends participated in the initiation of isoprene leading to the formation of MWNTs$g$-(BCB-PS- $b$-PI $)_{n}$. The DSC analysis of the samples showed that the surface grown PS has a higher glass-transition temperature, $T_{\mathrm{g}}\left(108.7^{\circ} \mathrm{C}\right)$ as compared to ordinary linear PS $\left(\sim 101{ }^{\circ} \mathrm{C}\right)$. Similarly, the surface grown PS- $b$-PI also had two distinct $T_{\mathrm{g}}$ values at -60.7 and at $104.2{ }^{\circ} \mathrm{C}$ corresponding to PI and PS, respectively (see the Supporting Information).

The presence of PS on the surface of MWNTs can be visualized through TEM analysis. A chloroform solution of MWNTs- $g$-(BCB-PS) ${ }_{n}$ was drop coated on a carbon grid. The TEM analysis of the MWNTs- $g$-(BCB-PS) ${ }_{n}$ showed the presence of amorphous material coated on the surface of the MWNTs (Figure 12). The layer thickness of grafted PS was as high as $30 \mathrm{~nm}$ on the surface of MWNTs- $g$-(BCB-PS) (Figure 12b). The coverage of polymer around the surface of MWNTs increases the volume and the surface area of MWNTs, substantially. For example, the MWNTs with 35 $\mathrm{nm}$ diameter covered with $24 \mathrm{~nm}$ polymer layer, as seen in Figure 12a, increased in volume by nearly half an order magnitude and nearly doubled in surface area. Such a large increase in the volume and the surface area, if covered by polymer homogeneously, would result in a MWNTs-polymer core-shell structure. However, the tubes are not covered homogeneously because of random initiator attachment. Although the actual length of the grafted PS could not be determined, a high apparent number-average molecular weight obtained from SEC of MWNTs- $g$-(BCB-PS) $)_{n}$ indicates that the grafted PS chain length is higher than PEO. These MWNT-grafted homopolymers and diblock copolymers bearing high molecular weight grafts can be viewed as novel MWNT-polymer brushes. They can also be used as a compatibilizer for MWNT-polymer nanocomposites.

\section{Conclusions}

Poly(ethylene oxide) and polystyrene were grown from the surface of MWNTs using living surface-initiated anionic polymerization. Using a "grafting to" strategy, MWNTs were covalently functionalized with 4-hydroxyethyl benzocyclobutene (BCB-EO) and 1-benzocylcobutene-1'-phenyleth- 
ylene (BCB-PE) through [4+2] cycloaddition. The presence of these precursor initiator moieties on the surface of MWNTs was confirmed by FT-IR, Raman spectroscopy, and TGA. Alkoxy anions and alkyllithium anions were generated from MWNTs- $g$-(BCB-EO) ${ }_{n}$ and MWNTs- $g$-(BCB-PE) $)_{n}$ using triphenylmethane and $s e c$-butyllithium for the polymerization of ethylene oxide and styrene in THF and benzene, respectively.

The initiation of ethylene oxide and styrene from the surface alkoxy and alkyl anions, respectively was found to be slow due to heterogeneous nature of the reaction. The initiator phenyl alkyllithium and the propagating polystyryl anions on the surface of MWNTs did not show their typical characteristic orange color because of the presence of the nanotubes. The polymerization of ethylene oxide from the surface of MWNTs- $g$-(BCB-EO) ${ }_{n}$ exhibited linear growth as indicated by the increase in monomer conversion and weight percent PEO on the MWNTs with increasing reaction time. The surface grown polymers were obtained in high conversion, forming MWNTs- $g$-(BCB-PEO) $)_{\mathrm{n}}$ and MWNTs$g$-(BCB-PS $)_{n}$ containing a small fraction of MWNTs $<1$ wt $\%$. Surface-grown polymer nanocomposites were char- acterized using FT-IR, ${ }^{1} \mathrm{H}$ NMR, Raman spectroscopy, DSC, TGA, and TEM. The TEM images showed the presence of thick layers of polymer around the surface. The MWNTs$g$-(BCB-PEO $)_{n}$ and MWNTs- $g$-(BCB-PS $)_{n}$ samples exhibit broad molecular weight distribution $\left(1.3<M_{\mathrm{w}} / M_{\mathrm{n}}<1.8\right)$ in SEC analysis. The formation of diblock copolymer, MWNTs- $g$-(BCB-PS- $b$-PI $)_{n}$ confirmed the living nature of these polymerizations.

Acknowledgment. This work was sponsored by the Division of Materials Science and Engineering, Office of Basic Energy Sciences, U.S. Department of Energy, under Contract DE-AC0500OR22725 with Oak Ridge National Laboratory, managed and operated by UT-Battelle, LLC.

Supporting Information Available: ${ }^{1} \mathrm{H}$ NMR of MWNTs- $g$ (BCB-PEO) , dissolution vials of PEO grafted MWNTs in water, TEMimages of MWNTs- $g$-(BCB-PS) $)_{n}$, Raman spectrum of MWNTs$g$-(BCB-EO) ${ }_{n}$, MWNTs- $g$-(BCB-PE) ${ }_{n}$, MWNTs- $g$-(BCB-PEO) ${ }_{n}$, MWNTs- $g$-(BCB-PS $)_{n}$, FT-IR of polystyrene grafted MWNTs, and DSC of MWNTs- $g$-(BCB-PS) $n_{n}$ and MWNTs- $g$-(BCB-PS-b-PI) ${ }_{n}$ $(\mathrm{PDF})$. This material is available free of charge via the Internet at http://www.acs.org.

CM801449T 